


\section{Tree diversity and its use by local communities in Buol District, Indonesia}

Subekti Rahayu, Betha Lusiana, Sacha Amaruzaman, Dienda Citasyari Putri Hendrawan and Sidiq Pambudi

Working paper no. 212 


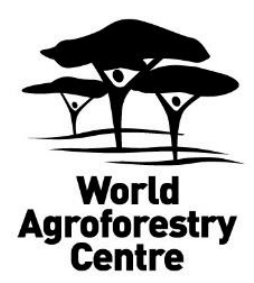

Citation: Rahayu S, Lusiana B, Amaruzaman S, Hendrawan DC, Pambudi S. 2015. Tree diversity and its use in Buol District, Indonesia. Working Paper 212. Bogor, Indonesia: World Agroforestry Centre (ICRAF) Southeast Asia Regional Program. DOI: http://dx.doi.org/10.5716/WP15723.PDF

Titles in the Working Paper Series aim to disseminate interim results on agroforestry research and practices and stimulate feedback from the scientific community. Other publication series from the World Agroforestry Centre include: agroforestry perspectives, technical manuals and occasional papers.

Published by the World Agroforestry Centre (ICRAF)

Southeast Asia Regional Program

PO Box 161, Bogor 16001

Indonesia

Tel: +62 2518625415

Fax: +62 2518625416

Email: icraf-indonesia@cgiar.org

Website: http://www.worldagroforestry.org/regions/southeast_asia

(C) World Agroforestry Centre 2015

Working Paper no. 212

\section{Disclaimer and copyright}

The views expressed in this publication are those of the author(s) and not necessarily those of the World Agroforestry Centre. Articles appearing in this publication may be quoted or reproduced without charge, provided the source is acknowledged. All images remain the sole property of their source and may not be used for any purpose without written permission of the source. 


\section{About the authors}

Subekti Rahayu is a biodiversity and carbon stock specialist at the World Agroforestry Centre. Her main areas of interest include biodiversity conservation, ecological restoration, forest ecology, bioindicators and agroforest ecology. She is a $\mathrm{PhD}$ candidate on ecological restoration strategies in Samboja Forest Research, East Kalimantan. She has a master degree in Tropical Biodiversity Conservation from Bogor Agriculture University. She holds a bachelor degree from the same university, majoring in Plant Protection. She has had experience with carbon-stock measurement at plot level since 1998 and delivering training on the subject to various institutions in Indonesia and to local communities in Indonesia and Viet Nam since 2002. Contact: s.rahayu@ @giar.org.

Betha Lusiana is a scientist with the World Agroforestry Centre (ICRAF) Indonesia program, where she leads the Ecological Modelling Unit. She also coordinates Smart Tree-Invest project activities in Indonesia. She has a $\mathrm{PhD}$ from Hohenheim University, Germany on the use of land-use-change models for natural research management. Her research focuses on assessing trade-offs between agricultural development, farmers' livelihoods and ecosystem services through the use of participatory, quantitative and/or model simulation approaches. In particular, she is interested in exploring and analysing use of such assessments for natural resource management. Contact:

b.lusiana@cgiar.org

Sacha Amaruzaman is an ecosystem services specialist at the World Agroforestry Centre in Bogor, Indonesia. He co-manages the Climate-Smart, Tree-Based, Co-investment in Adaptation and Mitigation in Asia (Smart Tree-Invest) project that operates in Indonesia, Viet Nam and the Philippines, with funding from the International Fund for Agricultural Development. He also carries out research under the CGIAR Research Program on Forests, Trees and Agroforestry, focusing mainly on ecosystem services' socioeconomic and institutional aspects. He obtained his master degree from Wageningen University, the Netherlands, majoring in Environmental Science. Contact: s.amaruzaman@cgiar.org

Dienda Citasyari Putri Hendrawan works at the World Agroforestry Centre in Bogor, Indonesia. She holds a bachelor degree from Institut Teknologi Bandung, majoring in Biology. She is working on agrobiodiversity systems in Bantaeng, Southeast Sulawesi and farmers' study group in Buol, Central Sulawesi. Contact: d.hendrawan@ cgiar.org

Sidiq Pambudi has a background in Biology, especially Landscape Ecology. He holds a bachelor degree in Science from Institut Teknologi Bandung. He has experience in above-ground carbon-stock estimation and biodiversity. He is working as a biophysics research assistant at the World Agroforestry Centre (ICRAF) Indonesia country office. Contact: s.pambudi@cgiar.org. 


\section{Abstract}

We assessed plot-level, tree diversity and various tree-based systems in Buol District, Central Sulawesi Province, Indonesia: complex, clove, coconut and cacao agroforests; monoculture teak; and undisturbed and disturbed forests. The objectives were to assess the tree diversity of various land-use systems, to identify what tree species were used by the communities and to identify land-use systems that provided habitats for forest tree species. The indicators for tree diversity were tree species' richness and tree composition collected using a Quick Biodiversity Survey approach. The use of tree diversity by the communities was assessed though focus-group discussions in three clusters of villages: the upstream and midstream of the Buol watershed; and the coastal area. We found that tree species' richness in complex agroforests was 70\% that of undisturbed forests. Only 5\% of forest species regenerated in complex agroforestry systems, mostly represented by pioneer species. This condition indicated that although the complex agroforests species' richness in Buol was relatively high, complex agroforests could not provide refugee for forest tree species. Therefore, conserving forests was very important for maintaining forest tree diversity in Buol. The remaining small patch of disturbed forest in Buol District was in a developing stage, indicated by higher species' richness at sapling stage. The community in Buol had low dependency on forest species and were more interested in growing domesticated, commercial species. Not so long ago, farmers still harvested rattan from the forest but at the time of study the rattan population had diminished, most likely caused by overharvesting.

\section{Keywords}

Agroforestry, Buol, Central Sulawesi, community forest use, tree diversity, tree species' richness 


\section{Acknowledgements}

This working paper is the preliminary synthesis of research undertaken during the first year of the Climate-smart, Tree-based Co-investment in Adaptation and Mitigation in Asia (Smart Tree-Invest) project in Buol District, Central Sulawesi Province, Indonesia carried out by the World Agroforestry Centre with support from the International Fund for Agricultural Development and the CGIAR Research Program on Forests, Trees and Agroforestry. We also extend our gratitude to Harti Ningsih and Ni'matul Khasanah for their contributions during field work and data analysis. 


\section{Contents}

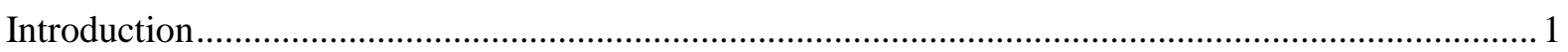

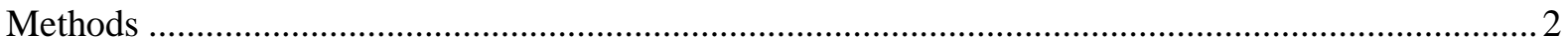

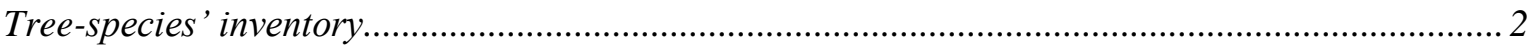

Local communities' perceptions of tree diversity and its functions ................................................ 3

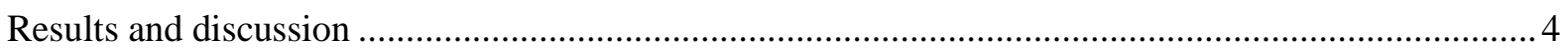

Current condition of tree-species' richness and diversity ................................................................. 4

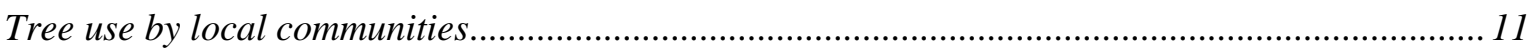

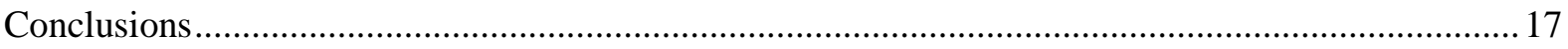

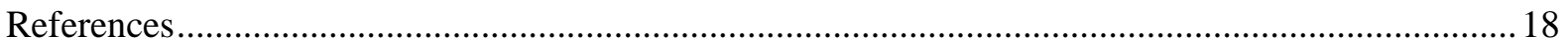

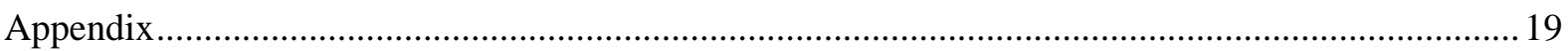

\section{List of Tables}

Table 1. Bray-Curtis similarity of seedlings across land-use systems .............................................. 9

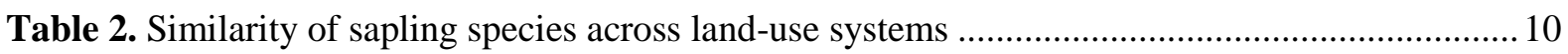

Table 3. Similarity of pole-stage species across land-use systems ................................................ 10

Table 4. Similarity of tree species across land-use systems ............................................................... 11

Table 5. Use of biodiversity by the upstream cluster in different land-use types, based on male and female perceptions

Table 6. Use of tree diversity by the upstream cluster in various land covers during normal and extreme conditions

Table 7. Use of biodiversity by the midstream cluster in various land-use types, based on male and female perceptions

Table 8. Use of tree diversity by the midstream cluster from various land covers during normal and extreme conditions

Table 9. Use of biodiversity by the coastal cluster in various land-cover types, based on female and male perceptions.

Table 10. Use of tree diversity by the coastal cluster in various land-cover types during normal and extreme conditions...... 


\section{List of Figures}

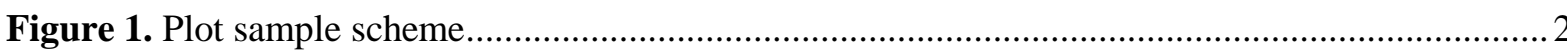

Figure 2. Species' accumulation curve of seedlings, saplings, poles and trees in each sub-plot of

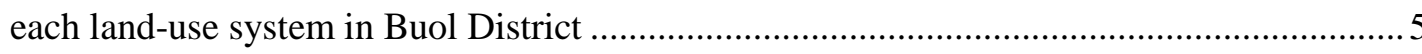

Figure 3. Species' accumulation curve of seedlings, saplings, poles and trees in each land-use system

Figure 4. Plot-level Shannon-Wiener Diversity Index in each vegetation stage of various land-uses in Buol......

Figure 5. Land-use-level Shannon-Wiener Diversity Index in each vegetation stage of various land-uses in Buol

Figure 6. Plot-level Shannon Evenness Index in each vegetation stage of the various land uses in Buol 8

Figure 7. Land-use-level Shannon Evenness Index in each vegetation stage of the various land uses in Buol 



\section{Introduction}

Biodiversity directly affects human life. Beyond production of food, fibre, fuel and income, biodiversity plays an important role in nutrient cycling, control of microclimates, regulation of hydrological process and abundance of undesirable microorganisms and detoxification of noxious chemicals (Altieri 1999). Biodiversity not only contributes to material welfare and livelihoods but also contributes to security, resiliency, social relations, freedom of choice and actions (MEA 2005). At the landscape scale, patches of ecosystems with high biodiversity are important in providing environmental services, such as being sources of seeds for regeneration, habitat refuges for plants and animals and regulation of climate and hydrology. A sustainable landscape ideally contains diverse ecosystems that provide various services for communities, compared to monoculture or simple systems that focus only on providing economic benefits and food.

Converting forests to other systems, particularly, human-made systems, such as agriculture or settlements, has a direct impact on biodiversity across a range of taxonomic groups and population sizes, or both, causing taxonomies and populations of the majority of species to decline (MEA 2005). Transmigration ${ }^{1}$ settlements and oil-palm plantations started in 1990s have driven land-use change in Buol. The forest area has declined substantially since 2000, mostly converted into complex agroforests, cacao agroforests and oil-palm plantations. The oil-palm plantation area increased by 55\% from 1995 to 2014, roughly covering 10\% of Buol District (Wijaya et al 2015). A land-use and land-cover analysis of the district indicated that since 1996 more than 14\% of undisturbed forest, which contained diverse tree species, was converted to other land use (Wijaya et al 2015). Besides the expansion of plantations, the development of transmigration settlements also potentially changed use of biodiversity by the community.

This study aimed to understand the condition of tree diversity in various land-use systems and the role of disturbed forests and complex agroforestry systems as habitat refuges for forest tree species. The specific objectives of the study were 1) to assess the tree diversity of various land-use systems in Buol District, including the use of trees by the communities; and 2) to identify land-use systems that provided habitat for tree diversity, both naturally grown and domesticated.

The hypotheses of the study were 1) complex agroforests and young secondary forests contained high tree diversity that allowed forest species to regenerate and play a role as habitat refuges; and 2) community dependency on naturally-grown species was low.

\footnotetext{
${ }^{1}$ Transmigration is Indonesia's program of moving people from the overcrowded islands of Java, Madura, Bali, and Lombok to the less crowded areas in other big islands of Sumatra, Kalimantan, Sulawesi, and Papua.
} 


\section{Methods}

\section{Tree-species' inventory}

\section{Field Survey}

A tree-diversity survey was conducted in seven land-use systems in Buol District: 1) disturbed forests;

2) sago forests; 3) complex agroforests; 4) cacao agroforests; 5) coconut agroforests; 6) clove agroforests; and 7) monoculture teak. Undisturbed forests in the immediate vicinity were sampled as a reference. In each land-use system, we sampled two to four replications of $20 \mathrm{~m}$ x $100 \mathrm{~m}$ nested plots that were further divided into subplots of 1) $5 \times 2 \mathrm{~m} \times 2 \mathrm{~m}$ to observe seedlings with diameters less than $5 \mathrm{~cm}$ and heights less than $2 \mathrm{~m}$;2) $5 \times 5 \mathrm{~m} \times 5 \mathrm{~m}$ to observe saplings with diameters less than 5 $\mathrm{cm}$ and more than $2 \mathrm{~m}$ height; 3) $5 \times 10 \mathrm{~m} \times 10 \mathrm{~m}$ to observe poles $5-10 \mathrm{~cm}$ in diameter and above 2 $\mathrm{m}$ in height; and 4) $5 \times 20 \mathrm{~m} \times 20 \mathrm{~m}$ to observe trees with more than $10 \mathrm{~cm}$ diameter (Figure 1).

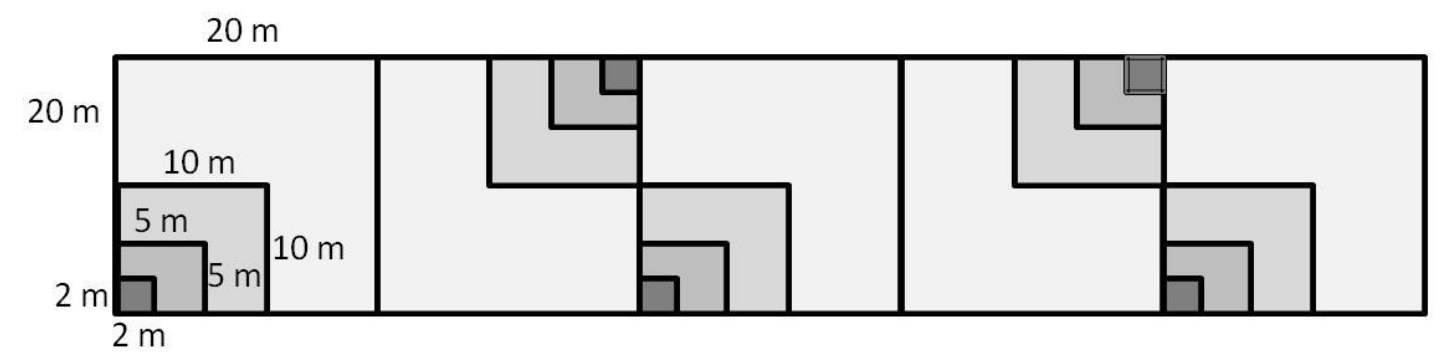

Figure 1. Plot sample scheme

The Herbarium Bogoriense in Bogor, West Java, Indonesia carried out a species' identification for all seedlings, saplings, poles and trees inside the plots using leaf specimens taken during a field survey. We measured the stem diameter at breast height $(\mathrm{DBH} \sim 1.3 \mathrm{~m})$ for all trees and poles.

\section{Data analysis}

We used four indicators of tree diversity: 1) rarefaction or accumulation species' curve, representing the tree-species' richness in each land-use system, or alpha diversity; 2) Shannon-Wiener Diversity Index of species in each plot and in each land-use system; and 3) Bray-Curtis Distance representing dissimilarity of species across different land-use systems, or beta diversity. Bray Curtis Distance measured dissimilarity, therefore, the similarity index used the following equation:

$1-B C=1-\frac{\sum_{i=1}^{S} \mid\left(n_{1 i}-n_{2 i} \mid\right)}{\sum_{i=1}^{S}\left(n_{1 i}+n_{2 i}\right)}$

Where: $\mathrm{BC}=$ Bray-Curtis dissimilarity, $\mathrm{S}=$ total species number in land use 1 and land use $2, \mathrm{n}_{1}=$ number of individual species $i$ in land use $1, n_{2}=$ number of individual species $i$ in land use 2. 
We used the Shannon-Wiener Diversity Index to derive Species Diversity and Species Evenness values to represent how close (equal) in numbers were the species in various land-use systems.

$H^{\prime}=-\sum_{i=1}^{\mathrm{s}} \mathrm{p}_{\mathrm{i}} \ln \mathrm{p}_{\mathrm{i}}$

Where: H' is Shannon-Wiener Diversity Index, while $\mathrm{p}_{\mathrm{i}}$ is the proportion of species i relative to the total number of species (S). If $\mathrm{H}^{\prime}<1$ categorized low diversity, $1<\mathrm{H}^{\prime}<1$ categorized medium diversity, $\mathrm{H}^{\prime}>1$ categorized high diversity.

Shannon Evenness was calculated by dividing $\mathrm{H}$ by $\mathrm{H}$ maximum (ln S):

$\mathrm{E}_{\mathrm{H}}=\frac{\mathrm{H}}{\operatorname{lnS}}$

Evenness assumes a value between 0 to 1 with 1 being complete evenness.

In our study, rarefaction curves were derived from both sub-plot and plot data (Figure 1). Thus, the results can represent both alpha diversity (sub-plot data) and beta diversity (plot data). The species' diversity referred to tree species in vegetation stages: seedlings, saplings, poles and trees.

We also calculated the similarity within the early stages of trees (seedlings and saplings) in similar land-use systems to measure survival ability at the seedling, sapling, pole and tree stages. Using the similarity index, we identified forest species found in non-forest land uses as an indicator of the ability of the non-forest land use to function as habitat for forest species.

\section{Local communities' perceptions of tree diversity and its functions}

We applied the Capacity Strengthening Approach to Vulnerability Assessment framework developed by the World Agroforestry Centre (Dewi et al 2013) to gather information on local communities' use of trees in their daily activities. Focus-group discussions were conducted in three clusters of villages located in the upstream (Lomuli and Kokobuka villages) and midstream (Air Terang and Boilan villages) of Buol Watershed and the coastal area (Lokodidi, Matinan and Taat villages) of Buol District (Lusiana et al 2015). Those areas are the cluster sites of an action research project, ClimateSmart, Tree-Based, Co-investment in Adaptation and Mitigation in Asia, carried out by the World Agroforestry Centre (ICRAF) in Buol District, 2014-2017. The upstream and midstream clusters were dominated by migrants from other parts of Indonesia, while the coastal cluster was dominated by people indigenous $t$ the area. In each cluster, the focus-group discussions were disaggregated by gender with each gender group (male and female) consisting of 5-7 participants. 


\section{Results and discussion}

\section{Current condition of tree-species' richness and diversity}

\section{Rarefaction curve}

A rarefaction or species' accumulation curve represents an estimation of the number of species that would be found if sampling efforts were increased or reduced. The analysis was applied to sub-plot (Figure 2) and plot (Figure 3) data. At the seedling stage, cacao agroforests represented the lowest number of species. Weeding most likely had an impact on seedling establishment in cacao agroforests. The number of species found in cacao agroforests gradually increased at the sapling, pole and tree stages.

The highest species' richness of seedlings was found in clove agroforests but the number of species significantly declined by the sapling stage, with clove agroforests then representing the lowest richness (Figure 2A and B). Similar to cacao agroforests, in clove agroforests local people carried out weeding periodically, particularly before harvesting, which contributed to the dynamic of species' richness at seedling and sapling stages.

We found that complex agroforests, disturbed forests and undisturbed forests had consistently high species' richness at seedling, sapling, pole and tree stages, ranging 9-11 species in 20 sampling units. Adding sampling units would potentially increase the number of species. We found that species' richness in complex agroforests was dominated by domesticated species, such as durian (Durio zibethinus), rambutan (Nephelium lappaceum), cacao (Theobroma cacao) and coconut (Cocos nucifera). 

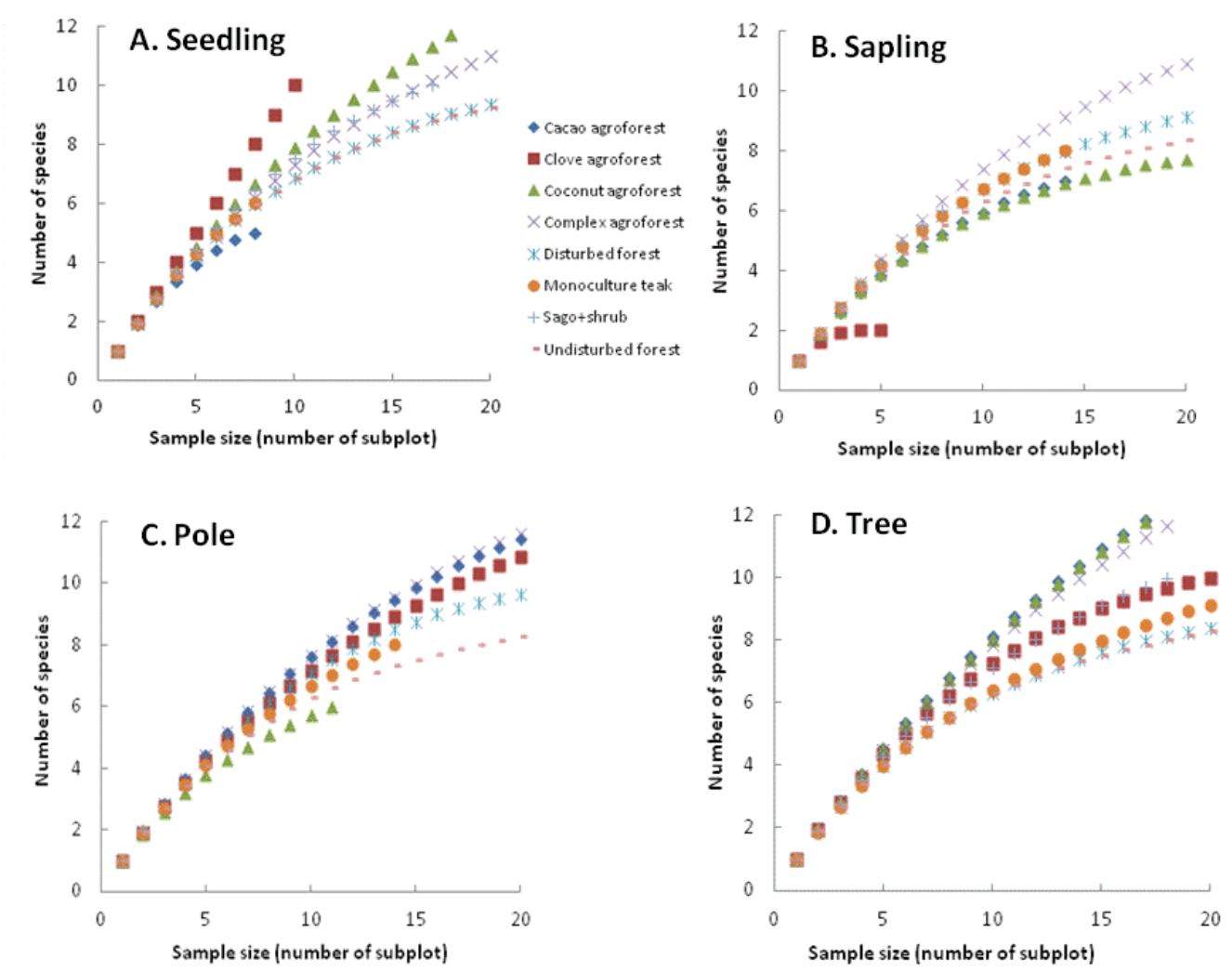

Figure 2. Species' accumulation curve of seedlings, saplings, poles and trees in each sub-plot of each land-use system in Buol District

A plot-level analysis showed similar results (Figure 3). Clove agroforests had relatively low species' richness of seedlings, saplings, poles and trees (Figure 3A, B, C, D), shown by the flat slope in the species' accumulation curve. This curve indicates that sampling more plots would not increase species' richness. Clove agroforests in Buol mostly consisted of monoculture species, representing only 2-3 tree species at seedling, and 3-4 at sapling, stages.

Low tree diversity, ranging 1-2 species, was also found in monoculture teak and sago forests. The species' richness in complex agroforests, disturbed forests and undisturbed forests was high, ranging 10-15 species in 20 sampling units. We found that the undisturbed forests had the highest species' richness at the seedling and pole stages and the disturbed forests had the highest at the sapling and tree stages. The high species' richness of all vegetation stages in the disturbed forests indicated that they are regenerating. The species' richness in complex agroforests decreased at pole and tree stages to less than nine species in each stage. Intensive weeding carried out in the complex agroforests mainly contributed to the loss of species, particularly, for naturally-regenerated tree species that were not being used. Farmers who managed perennial, crop-based agroforests only maintained domesticated tree species for the additional benefits of shade and fruit. 

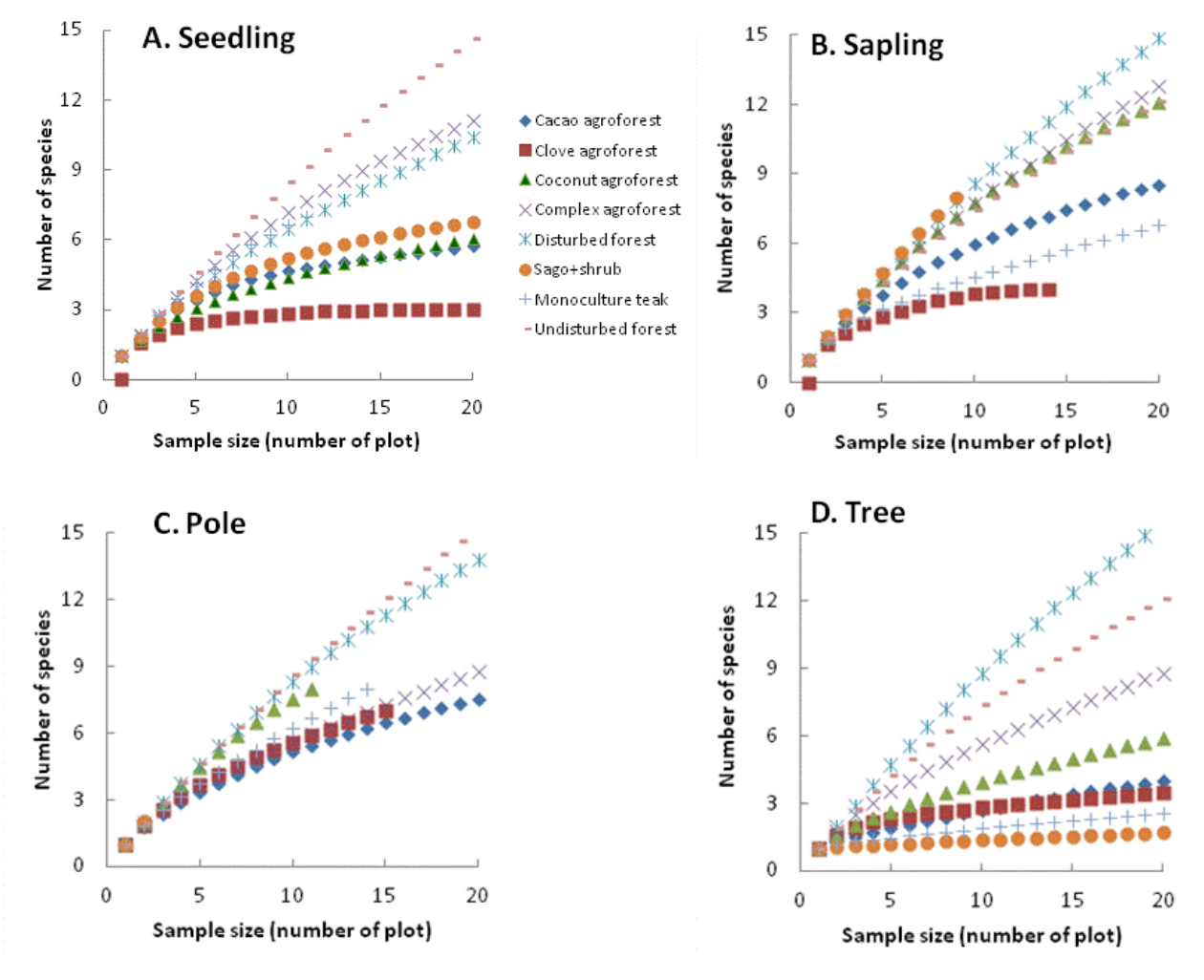

Figure 3. Species' accumulation curve of seedlings, saplings, poles and trees in each land-use system

\section{Shannon-Wiener Index}

The Shannon-Wiener Index (H') was used to quantify species' diversity both at plot and land-use levels. From a plot-level analysis, we found that tree-species' diversity was mostly in the medium range $\left(1<\mathrm{H}^{\prime}<3\right)$ value, except for the sapling stage in clove agroforests and pole stage in sago systems, which represented the low range value of $\mathrm{H}^{\prime}<1$ (Figure 4).

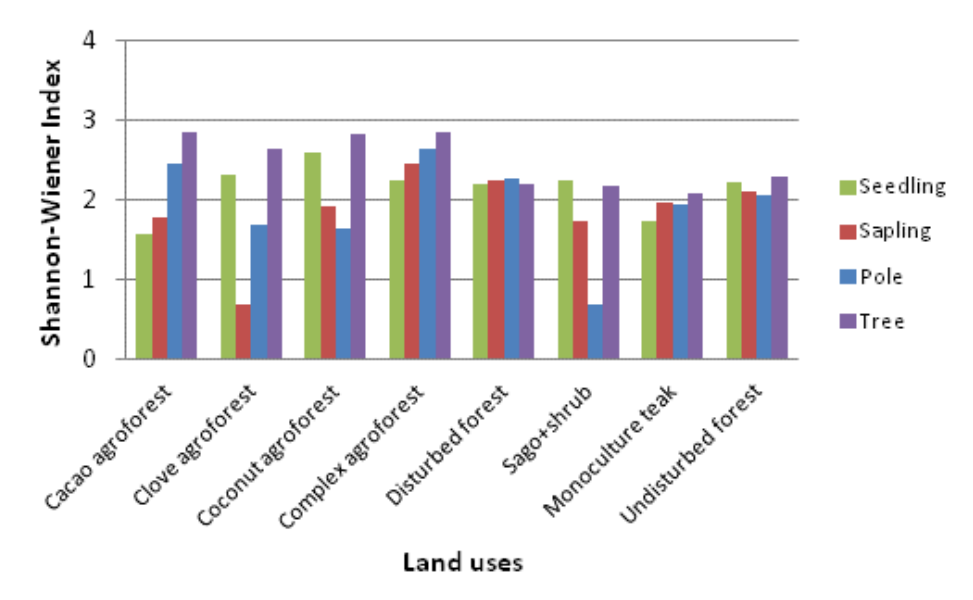

Figure 4. Plot-level Shannon-Wiener Diversity Index in each vegetation stage of various land-uses in Buol 
Species' diversity was relatively similar between the disturbed and undisturbed forests and slightly higher in the cacao agroforests. Intensive weeding and species' selection by farmers resulted in low species' diversity of seedlings and saplings in the survey, consequently, we can predict that the diversity of poles and trees will decline in the future. Higher diversity in all vegetation stages can be found in complex agroforests and teak monoculture.

Farmers allowed other tree species to regenerate in teak plantations-which were commonly managed as monoculture - up to the tree stage. This interesting management resulted in higher tree diversity for teak plantations.

At the land-use level, high species' diversity of H’>3 was found in disturbed forests, particularly at the sapling and pole stages (Figure 5). This was influenced by the regeneration of several pioneer species when disturbance occurred about 5-10 years prior to the survey. As the forest cover increased, the number of seedlings of the pioneer species declined and were replaced by the seedlings of shadetolerant species. Low species' diversity was mostly found at the tree stage of cacao agroforests, clove agroforests, sago forests and monoculture teak, and at the seedling stage of cacao agroforests.

Complex agroforests and undisturbed forests had a similar pattern of species' diversity balance across all vegetation stages.

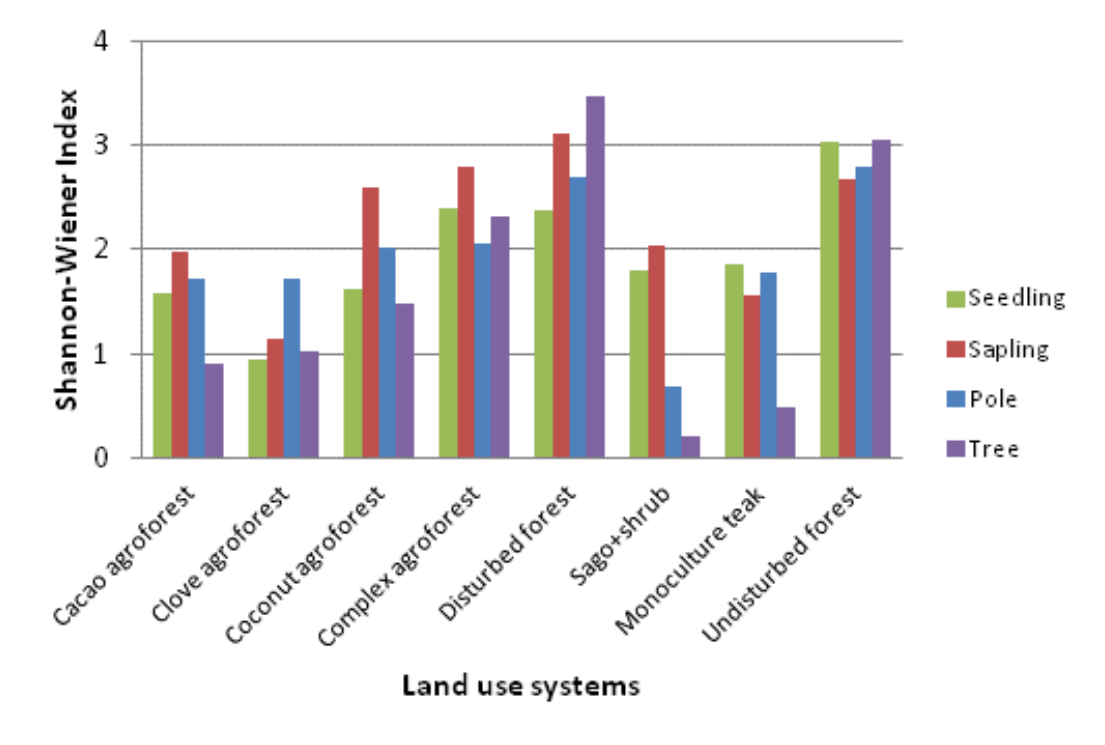

Figure 5. Land-use-level Shannon-Wiener Diversity Index in each vegetation stage of various land-uses in Buol

The Shannon Evenness Index (J) was used to quantify the evenness of species both at plot and landuse levels. From the plot-level calculation, we found a high evenness index of $\mathrm{J}>0.9$ for all vegetation stages in the various land uses (Figure 6). This result indicated that the number of tree species were evenly distributed in each sub-plot sample. A low evenness index at the pole stage of clove agroforests indicated that there were several found only at the specific sub-plot within the plot samples. 


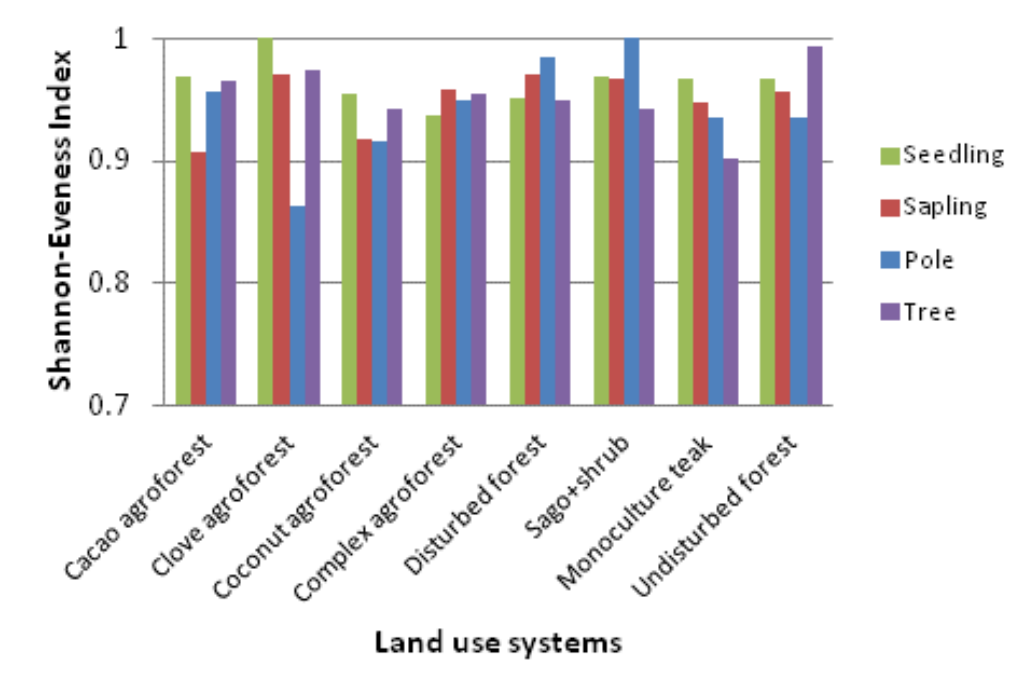

Figure 6. Plot-level Shannon Evenness Index in each vegetation stage of the various land uses in Buol

The evenness index decreased at land-use level (Figure 7). This was not surprising as it meant that some species tended to grow only in specific land uses. For example, sago will tend to be found in sago systems and nowhere else and teak can only be found in teak systems. We found low evenness (J $<0.2$ ) for the tree stage in cacao agroforests, sago, and monoculture teak owing to low populations of species at the tree stage.

A higher evenness index was found in all vegetation stages in complex agroforests, disturbed forests and undisturbed forests. This indicated that all vegetation stages were distributed in more sample plots in those three land-use systems compared to other systems, such as cacao agroforests, sago and monoculture teak. We only found a sample of young teak that had just reached the pole stage, up to 9 years-old. 


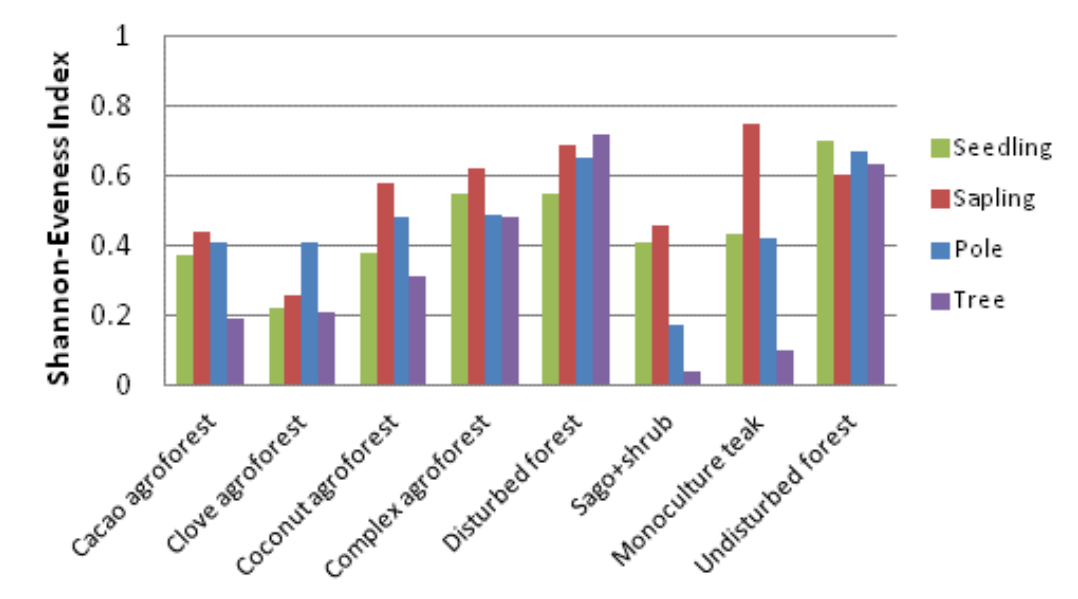

Land use systems

Figure 7. Land-use-level Shannon Evenness Index in each vegetation stage of the various land uses in Buol

\section{Tree-species' similarity}

At the seedling stage, the similarity of species between forests (disturbed and undisturbed) and other land-use systems was low. The complex agroforests and disturbed forests shared the highest similarity of 5\% and the species Tabernaemontana orientalis and Barringtonia tryscila. This finding indicated that the opportunity of forest species to regenerate in agroforestry systems in Buol District was low. The highest similarity at the seedling stage was found between clove and coconut agroforests at $35 \%$ (Table 1), which shared guava (Psidium guajava), Tabernaemontana orientalis, clove (Syzygium aromaticum) and coconut (Cocos nucifera).

Table 1. Bray-Curtis similarity of seedlings across land-use systems

\begin{tabular}{|l|l|l|l|l|l|l|l|l|}
\hline Land use & Cacao & Clove & Coconut & Complex & Disturbed & Sago & Teak & Un-disturbed \\
\hline Cacao & 1 & & & & & \\
\hline Clove & 0.0 & 1 & & & & \\
\hline Coconut & 0.02 & 0.35 & 1 & & & & \\
\hline Complex & 0.07 & 0.30 & 0.21 & 1 & 1 & & \\
\hline Disturbed & 0.05 & 0.0 & 0.03 & 0.05 & 0.0 & & \\
\hline Sago & 0.0 & 0.10 & 0.0 & 0.0 & 0.07 & 1 \\
\hline Teak & 0.12 & 0.18 & 0.02 & 0.19 & 0.07 & 0.03 \\
\hline Undisturbed & 0.0 & 0.03 & 0.0 & 0.0 & 0.08 & 1 \\
\hline
\end{tabular}

At the seedling stage, Tabernaemontana orientalis was the most common species in all plot samples. The species' similarity for the seedling stage between disturbed and undisturbed forest was $8 \%$, with three species - Ganua motleyana, Dracontomelon dao and Polyalthia lateriflora - commonly found in both systems. 
At the sapling stage, the species' similarity between the forests (disturbed and undisturbed) and other land-use systems in Buol was low, with the highest value of only 5\% similarity with cacao agroforests. The highest species' similarity was found between monoculture teak and cacao agroforests, at $40 \%$ similarity (Table 2 ).

Monoculture teak and cacao agroforests shared Piper aduncum and Saurauia tristyla at the sapling stage. P. aduncum was a pioneer species commonly found in open and managed areas of most landuse systems in Indonesia. The species' similarity between disturbed and undisturbed forests at the sapling stage was $20 \%$, higher than the seedling stage. The disturbed and undisturbed forests shared the similarity of Aglaia odoratissima, Dracontomelon dao, Licuala spinosa, Macaranga gigantea and Spathiostemon javensis.

Table 2. Similarity of sapling species across land-use systems

\begin{tabular}{|c|c|c|c|c|c|c|c|c|}
\hline Land use & Cacao & Clove & Coconut & Complex & Disturbed & Sago & Teak & Undisturbed \\
\hline Cacao & 1 & & & & & & & \\
\hline Clove & 0.09 & 1 & & & & & & \\
\hline Coconut & 0.21 & 0.08 & 1 & & & & & \\
\hline Complex & 0.15 & 0.12 & 0.23 & 1 & & & & \\
\hline Disturbed & 0.05 & 0.0 & 0.02 & 0.04 & 1 & & & \\
\hline Sago & 0.05 & 0.0 & 0.09 & 0.10 & 0.03 & 1 & & \\
\hline Teak & 0.40 & 0.1 & 0.13 & 0.13 & 0.05 & 0.0 & 1 & \\
\hline Undisturbed & 0.0 & 0.0 & 0.02 & 0.02 & 0.20 & 0.0 & 0.0 & 1 \\
\hline
\end{tabular}

At the pole stage, species' similarity between forests (disturbed and undisturbed) was $7 \%$. This result was higher than the seedling and sapling stages. The highest similarity was found between complex and cacao agroforests at 39\% (Table 3), with the domesticated species of rambutan (Nephelium lappaceum), durian (Durio zibethinus), cacao (Theobroma cacao) and Ficus septica being shared.

Table 3. Similarity of pole-stage species across land-use systems

\begin{tabular}{|c|c|c|c|c|c|c|c|c|}
\hline Land use & Cacao & Clove & Coconut & Complex & Disturbed & Sago & Teak & Undisturbed \\
\hline Cacao & 1 & & & & & & & \\
\hline Clove & 0.05 & 1 & & & & & & \\
\hline Coconut & 0.10 & 0.23 & 1 & & & & & \\
\hline Complex & 0.39 & 0.09 & 0.03 & 1 & & & & \\
\hline Disturbed & 0.03 & 0.05 & 0.0 & 0.07 & 1 & & & \\
\hline Sago & 0.0 & 0.0 & 0.0 & 0.04 & 0.0 & 1 & & \\
\hline Teak & 0.09 & 0.07 & 0.08 & 0.12 & 0.0 & 0.0 & 1 & \\
\hline Undisturbed & 0.0 & 0.0 & 0.0 & 0.02 & 0.04 & 0.07 & 0.0 & 1 \\
\hline
\end{tabular}


At the tree stage, the highest similarity of 53\% could be found between the complex and cacao agroforests (Table 4). The species' similarity between forests (disturbed and undisturbed) and other land-use systems was low, with the highest similarity of $4 \%$ found between the disturbed forests and complex agroforests.

The complex and cacao agroforests shared domesticated species-such as Artocarpus heterophyllus, Nephelium lappaceum, Durio zibethinus, Cocos nucifera, Mangifera indica, Theobroma cacao and Ceiba pentandra - and naturally-grown species, such as Aglaia sp, Kleinhova hospita and Artocarpus lanceifolius. At this stage, the species' similarity between disturbed forests and undisturbed forests was low at 9\%, with Dracontomelon dao as the shared species.

Table 4. Similarity of tree species across land-use systems

\begin{tabular}{|c|c|c|c|c|c|c|c|c|}
\hline Land use & Cacao & Clove & Coconut & Complex & Disturbed & Sago & Teak & Undisturbed \\
\hline Cacao & 1 & & & & & & & \\
\hline Clove & 0.09 & 1 & & & & & & \\
\hline Coconut & 0.10 & 0.30 & 1 & & & & & \\
\hline Complex & 0.53 & 0.17 & 0.33 & 1 & & & & \\
\hline Disturbed & 0.02 & 0.0 & 0.01 & 0.04 & 1 & & & \\
\hline Sago & 0.01 & 0.0 & 0.02 & 0.02 & 0.00 & 1 & & \\
\hline Teak & 0.06 & 0.01 & 0.09 & 0.07 & 0.03 & 0.0 & 1 & \\
\hline Undisturbed & 0.01 & 0.0 & 0.0 & 0.03 & 0.09 & 0.00 & 0.00 & 1 \\
\hline
\end{tabular}

\section{Tree use by local communities}

Migrant communities from Java and South Sulawesi (Bugis people), Bali and Lombok cultivated horticulture (annual crops), such as vegetables and pulses, in dry land converted from forests or shrubs. They also managed rice fields, converted from swamp sago forests, as their main source of income. Employees of oil-palm plantations were the potential market for horticultural products. Gender influence on perceptions of use of biodiversity can be seen in Table 5. 
Table 5. Use of biodiversity by the upstream cluster in different land-use types, based on male and female perceptions

\begin{tabular}{|c|c|c|c|c|c|c|c|}
\hline Land cover & $\begin{array}{l}\text { Building } \\
\text { material }\end{array}$ & Firewood & Fencing & Food & Income & Medicine & Others \\
\hline Degraded forest & 車 & ஜ் & - & & & $\bullet$ & $\dot{1}$ \\
\hline Oil-palm plantation & - & - & - & & & - & - \\
\hline Dry land & - & - & - & & & • & - \\
\hline Rice field & - & - & - & & & ํㅜ & 㟗 \\
\hline Cacao agroforest & - & - & - & & & ก & - \\
\hline Swamp & - & - & - & & - & - & - \\
\hline Shrub & in & in & ஸ் & & •. & - & - \\
\hline
\end{tabular}

In Buol, long dry spells occurred in 1998 and 2009. The droughts drew pigs and monkeys from the forests to the villages, becoming pests in the agricultural systems. At those times, the farmers were not able to grow rice, vegetables, pulses and other annual crops for food or income.

Local migrants (Buol people) used forests as sources of timber, such as 'lingua' (Pterocarpus indicus), 'palapi' (Heritiera sp.), 'cempaga' (Elmerillia sp.), meranti (Shorea sp.), 'nantu' (Palaquium sp.), 'bayur' (Pterospermum celebicum), 'gaharu' (Gyrinops versteghii), dammar (Agathis sp.), rattan and 'aren' (Arenga pinnata). The biodiversity that the smallholders used during normal and extreme conditions was relatively similar, particularly for perennial plants, but slightly different for annual crops that were more vulnerable to disasters and pests (Table 6). The extreme conditions that had occurred in the cluster included droughts, floods, prolonged rains, pests and diseases. 
Table 6. Use of tree diversity by the upstream cluster in various land covers during normal and extreme conditions

\begin{tabular}{|c|c|c|c|c|}
\hline \multirow[t]{2}{*}{ Land cover } & \multicolumn{2}{|c|}{ Normal condition } & \multicolumn{2}{|c|}{ Extreme condition } \\
\hline & & iீ & & ஜீ \\
\hline $\begin{array}{l}\text { Degraded } \\
\text { forest }\end{array}$ & $\begin{array}{l}\text { - Timber (building } \\
\text { material) } \\
\text { - Market items (income) } \\
\text { - Fruit }\end{array}$ & $\begin{array}{l}\text { - Timber (building } \\
\text { material) } \\
\text { - Market items (income) } \\
\text { - Firewood }\end{array}$ & $\begin{array}{l}\text { - Timber (building } \\
\text { material) } \\
\text { - Market items (income) } \\
\text { - Fruit }\end{array}$ & $\begin{array}{l}\text { - Timber (building } \\
\text { material) } \\
\text { - Market items (income) } \\
\text { - Firewood }\end{array}$ \\
\hline $\begin{array}{l}\text { Oil-palm } \\
\text { plantation }\end{array}$ & - Market items (income) & $\begin{array}{l}\text { - Market items (income) } \\
\text { - Fertilizer } \\
\text { - Erosion prevention }\end{array}$ & - Market items (income) & $\begin{array}{l}\text { - Market items (income) } \\
\text { - Fertilizer } \\
\text { - Erosion prevention }\end{array}$ \\
\hline Dry land & - & - Fruit & - & - \\
\hline Rice field & - & - & - & - \\
\hline $\begin{array}{l}\text { Cacao } \\
\text { agroforest }\end{array}$ & $\begin{array}{l}\text { - Fruit trees } \\
\text { - Timber (building } \\
\text { material) } \\
\text { - Market items (income) }\end{array}$ & $\begin{array}{l}\text { - Fruit trees } \\
\text { - Market items (income) } \\
\text { - Shade trees }\end{array}$ & $\begin{array}{l}\text { - Fruit trees } \\
\text { - Timber (building } \\
\text { material) } \\
\text { - Market items (income) }\end{array}$ & $\begin{array}{l}\text { - Fruit trees } \\
\text { - Market items (income) } \\
\text { - Shade trees }\end{array}$ \\
\hline Swamp & $\begin{array}{l}\text { - Food } \\
\text { - Fencing }\end{array}$ & - & $\begin{array}{l}\text { - Food } \\
\text { - Fencing }\end{array}$ & - \\
\hline Shrub & $\begin{array}{l}\text { - Timber } \\
\text { - Firewood } \\
\text { - Fruit }\end{array}$ & - & $\begin{array}{l}\text { - Timber } \\
\text { - Firewood }\end{array}$ & - \\
\hline
\end{tabular}

The community gathered timber, such as 'kayu gempol' (Neonauclea sp.), 'kayu mas' (Anthocephalus chinensis), 'kayu kenanga' (Cananga odorata), aren (Arenga pinnata) and firewood, such as 'kayu tutup' (Mallotus sp.), 'kayu sirih' (Piper aduncum) and 'gamal' (Gliricidia sepium) from swamp forests and shrub land. Migrants from Java, Madura, Bali and Lombok preferred to enrich their land with fruit trees, such as rambutan (Nephelium lappaceum), 'langsat' (Lansium domesticum), durian (Durio zibethinus), coconut (Cocos nucifera), rose apple (Syzygium aqueum), 'jengkol' (Archidendron jiringa), 'petai' (Parkia speciosa); timber tree species, such as teak (Tectona grandis), nantu (Palaquium sp.), cempaga (Elmerilliia sp.) and shade tree species, such as gamal (Gliricidia sepium) and 'dadap' (Erythrina sp.), in cacao agroforests. Women used tubers that they collected from dry land as a source of food.

The establishment of oil-palm plantations in the upstream cluster had provided other livelihoods' options for both local migrants (Buol people) and transmigrants from Java, Sulawesi, Bali, Madura and Lombok. They were employed as day-labourers and as food suppliers.

Migrant communities in the midstream cluster were highly dependent on agricultural products from rice fields because forest products, particularly, high-value timber, were no longer available.

However, the communities mentioned that they still used trees and other products from forests and other land-use systems (Table 7). 
Table 7. Use of biodiversity by the midstream cluster in various land-use types, based on male and female perceptions

\begin{tabular}{|c|c|c|c|c|c|c|c|}
\hline Land cover & $\begin{array}{l}\text { Building } \\
\text { material }\end{array}$ & Firewood & Food & Income & $\begin{array}{c}\text { Erosion } \\
\text { prevention }\end{array}$ & Medicine & Others \\
\hline Forest & & - & & & - & & \\
\hline Shrub & & $\dot{m}$ & & & - & - & - \\
\hline Seasonal crops & & - & & 0 & - & $\dot{\operatorname{m}}$ & - \\
\hline Rice field & & - & & - & - & "் & ก \\
\hline $\begin{array}{l}\text { Oil-palm } \\
\text { plantation }\end{array}$ & & - & & & $\dot{n}$ & - & - \\
\hline Swamp & - & - & & 舟 & - & - & - \\
\hline
\end{tabular}

Note: Others = decorative plants, bird food and fodder;

The use of biodiversity by men and women in the midstream cluster was relatively similar during normal and extreme conditions, as shown in Table 8.

Table 8. Use of tree diversity by the midstream cluster from various land covers during normal and extreme conditions

\begin{tabular}{|c|c|c|c|c|}
\hline \multirow[t]{2}{*}{ Land cover } & \multicolumn{2}{|c|}{ Normal condition } & \multicolumn{2}{|c|}{ Extreme condition } \\
\hline & & กீ & & กั \\
\hline $\begin{array}{l}\text { Degraded } \\
\text { forest }\end{array}$ & $\begin{array}{l}\text { - Timber (building } \\
\text { material) } \\
\text { - Market items } \\
\text { (income) } \\
\text { - Fruits }\end{array}$ & $\begin{array}{l}\text { - Timber (building } \\
\text { material) } \\
\text { - Market items } \\
\text { (income) } \\
\text { - Firewood } \\
\text { - Fruits }\end{array}$ & $\begin{array}{l}\text { - Timber (building } \\
\text { material) } \\
\text { - Market items } \\
\text { (income) } \\
\text { - Fruits }\end{array}$ & $\begin{array}{ll}\text { - } & \text { Timber (building } \\
\text { material) } \\
\text { - } \\
\text { - } \text { (incorket items } \\
\text { - Firewood } \\
\text { - Fruits }\end{array}$ \\
\hline $\begin{array}{l}\text { Oil-palm } \\
\text { plantation }\end{array}$ & $\begin{array}{l}\text { - Market items } \\
\text { (income) }\end{array}$ & $\begin{array}{l}\text { - Market items } \\
\text { (income) }\end{array}$ & $\begin{array}{l}\text { - Market items } \\
\text { (income) }\end{array}$ & $\begin{array}{l}\text { - Market items } \\
\text { (income) }\end{array}$ \\
\hline Dry land & $\begin{array}{l}\text { - } \text { Timber (building } \\
\text { material) } \\
\text { - } \text { Firewood } \\
\text { - } \text { Fruit trees }\end{array}$ & - Fruit trees & $\begin{array}{l}\text { - } \text { Timber (building } \\
\text { material) } \\
\text { - Firewood } \\
\text { - } \text { Fruit trees }\end{array}$ & - Fruit trees \\
\hline Rice field & - Food & - & - Food & - \\
\hline $\begin{array}{l}\text { Cacao } \\
\text { agroforest }\end{array}$ & 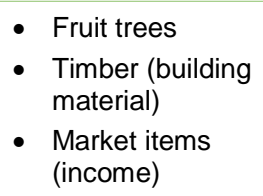 & $\begin{array}{ll}\text { - } & \text { Fruit trees } \\
\text { - } & \text { Market items } \\
\text { (income) }\end{array}$ & $\begin{array}{l}\text { - } \text { Fruit trees } \\
\text { - Timber (building } \\
\text { material) } \\
\text { - } \text { Market items } \\
\text { (income) }\end{array}$ & 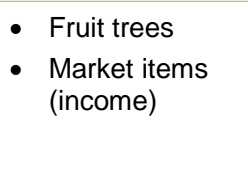 \\
\hline Swamp & $\begin{array}{l}\text { - Timber (building } \\
\text { material) } \\
\text { - Market items } \\
\text { (income) } \\
\text { - Food }\end{array}$ & - & $\begin{array}{l}\text { - Timber (building } \\
\text { material) } \\
\text { - Food }\end{array}$ & - \\
\hline Shrub & $\begin{array}{l}\text { - Food } \\
\text { - Fruit trees }\end{array}$ & $\begin{array}{l}\text { - Timber (building } \\
\text { material) }\end{array}$ & $\begin{array}{l}\text { - Food } \\
\text { - Fruit trees }\end{array}$ & $\begin{array}{l}\text { - Timber (building } \\
\text { material) }\end{array}$ \\
\hline
\end{tabular}




\begin{tabular}{|c|c|c|c|c|}
\hline \multirow[t]{2}{*}{ Land cover } & \multicolumn{2}{|c|}{ Normal condition } & \multicolumn{2}{|c|}{ Extreme condition } \\
\hline & $\begin{array}{l}\text { - Timber (building } \\
\text { material) }\end{array}$ & $\begin{array}{l}\text { - } \text { Shade trees } \\
\text { - } \text { Fruit } \\
\text { - } \text { Market items } \\
\text { (income) }\end{array}$ & $\begin{array}{l}\text { - } \quad \text { Timber (building } \\
\text { material) }\end{array}$ & $\begin{array}{l}\text { - } \text { Shading trees } \\
\text { - } \text { Fruit } \\
\text { - } \text { Market items } \\
\text { (income) }\end{array}$ \\
\hline
\end{tabular}

The community in the midstream cluster used trees from secondary forests in the immediate vicinity for building materials, such as Neonauclea sp., Anthocephalus chinensis and Alstonia scholaris. From around 2010 , the communities gradually abandoned their cacao agroforests owing to the seemingly unsolvable problem of pests and diseases.

The local people of the coastal area depended on agricultural products, both from rice fields and perennial crops. They managed agroforestry systems, such as clove, coconut and cacao. The mangrove systems along the coast were perceived to provide protection from waves. The mangroves, however, have suffered from deforestation, their area is diminishing and coastal abrasion increasing. The community also perceived other benefits from mangroves, such as habitat for fish and shrimps, which provided food and income (Table 9).

Table 9. Use of biodiversity by the coastal cluster in various land-cover types, based on female and male perceptions

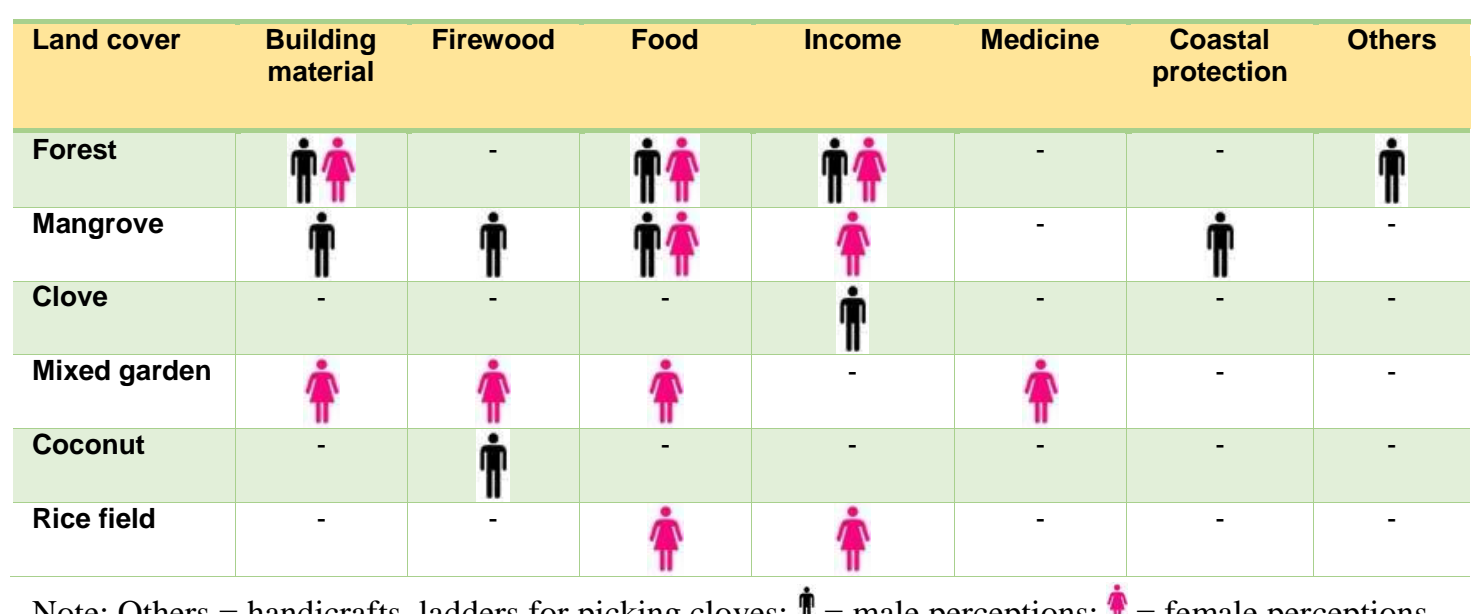

Compared to the upstream and midstream clusters, the coastal communities had different strategies for using tree diversity under extreme conditions (Table 10). Coastal people changed their livelihoods' strategies from land-based to marine-based. 
Table 10. Use of tree diversity by the coastal cluster in various land-cover types during normal and extreme conditions

\begin{tabular}{|c|c|c|c|c|}
\hline \multirow[t]{2}{*}{ Land cover } & \multicolumn{2}{|c|}{ Normal condition } & \multicolumn{2}{|c|}{ Extreme condition } \\
\hline & & & & \\
\hline $\begin{array}{l}\text { Degraded } \\
\text { forest }\end{array}$ & $\begin{array}{l}\text { - Timber (building } \\
\text { material) } \\
\text { - Market items } \\
\text { (income) } \\
\text { - Fruits } \\
\text { - Others }\end{array}$ & $\begin{array}{ll}\text { - } & \text { Timber (building } \\
\text { material) } \\
\text { - } \text { Food } \\
\text { - } \text { Market items } \\
\text { (income) }\end{array}$ & $\begin{array}{ll}\text { - } & \text { Timber (building } \\
\text { material) } \\
\text { - } \text { Rattan } \\
\text { - } \text { Market items } \\
\text { (income) } \\
\text { - } \text { Fruits } \\
\text { - Others }\end{array}$ & $\begin{array}{ll}\text { - } & \text { Timber (building } \\
& \text { material) } \\
\text { - } & \text { Food } \\
\text { - } & \text { Market items } \\
& \text { (income) }\end{array}$ \\
\hline Mangrove & $\begin{array}{l}\text { - Timber (building } \\
\text { material) } \\
\text { - Others }\end{array}$ & $\begin{array}{l}\text { - Timber (building } \\
\text { material) }\end{array}$ & $\begin{array}{l}\text { - Timber (building } \\
\text { material) } \\
\text { - Others }\end{array}$ & $\begin{array}{l}\text { - Timber (building } \\
\text { material) }\end{array}$ \\
\hline Mixed garden & $\begin{array}{l}\text { - } \text { Timber (building } \\
\text { material) } \\
\text { - } \text { Fruit } \\
\text { - } \text { Market items } \\
\text { (income) }\end{array}$ & $\begin{array}{ll}\text { - } & \text { Timber (building } \\
\text { material) } \\
\text { - } & \text { Fruits } \\
\text { - } & \text { Market items } \\
\text { (income) }\end{array}$ & $\begin{array}{ll}\text { - } & \text { Timber (building } \\
\text { material) } \\
\text { - } \text { Fruits } \\
\text { - } \text { Market items } \\
\text { (income) }\end{array}$ & $\begin{array}{ll}\text { - } & \text { Timber (building } \\
\text { material) } \\
\text { - } \text { Fruit } \\
\text { - } \text { Market items } \\
\text { (income) }\end{array}$ \\
\hline Rice field & - & - & - & - \\
\hline Coconut & $\begin{array}{ll}\text { - } & \text { Market items } \\
\text { (income) } \\
\text { - } \\
\text { - } & \text { Fruit } \\
\text { - } & \text { irewood }\end{array}$ & - & $\begin{array}{ll}\text { - } & \text { Market items } \\
\text { (income) } \\
\text { - } & \text { Fruit } \\
\text { - } & \text { Firewood }\end{array}$ & - \\
\hline Clove & $\begin{array}{l}\text { - Market items } \\
\text { (income) }\end{array}$ & - & $\begin{array}{l}\text { - Market items } \\
\text { (income) }\end{array}$ & - \\
\hline
\end{tabular}

Extreme conditions of frequent occurrence in the upstream cluster provoked the communities to differing responses. Migrants from Java, Madura, Bali and Lombok, in particular, enriched their agricultural land with more species, particularly for domestic consumption. Local Buol migrants used the remaining forests to collect aren, rattan and timber and used sago as a food source.

In the midstream cluster, where agricultural production was the main livelihood, pests and diseases had a heavy impact on the production of rice, cacao and cloves. Unfortunately, the farmers had not developed any satisfactory measures for controlling pests and diseases, except for wealthier farmers, who could afford pesticides. Most farmers would instead replant with other crops if they had the financial capital or could borrow (money, seedlings, production inputs) from friends, loan agents and store owners. Farmers without access to capital or loans would fallow their rice fields, labour for wealthier farmers or sell banana and cassava chips as their livelihoods' alternatives.

In the coastal area, rice, cloves and fisheries were the main livelihoods during normal years. During extreme conditions, the main option was to work as labour in urban settlements, such as the district capital.

The communities in all clusters were aware that availability of timber in their immediate forests had significantly decreased in recent years. Activities to conserve or plant forest timber species on their managed land, however, were not yet an option since they had no capacity for domesticating such species. 


\section{Conclusions}

Tree diversity in Buol District, Central Sulawesi in all land-use systems was dominated by domesticated species with high economic value, with the exception of disturbed forests. The communities had low dependency on trees, particularly on naturally-grown trees. Both the migrant communities from Java, Madura, Bali and Lombok and the people of Buol origin showed an interest in managing agro-biodiversity that consisted of annual and perennial crops, mainly as sources of income. Dependency on naturally-grown tree species as a source of income was low, as timber and rattan were no longer found in the immediate forests owing to over-harvesting in the past. Sago and Arenga pinata systems were potential land uses with high tree diversity, also for food provisioning and sources of income. However, these species had not been optimally used.

Tree-species' richness in disturbed forests in Buol was similar to undisturbed forests but the species' composition differed significantly. Only $20 \%$ of pole-stage species in disturbed forests was similar to those in undisturbed forests. Disturbed forests consisted of pioneer species, indicating that the forests are regenerating. Complex agroforests and disturbed forests could potentially provide habitat for forest tree species.

Farmer-managed systems of cacao, clove and coconut agroforests and teak in Buol cannot function as refuges for forest tree species. They are, unsurprisingly, dominated by domesticated economic species. Complex agroforests and disturbed forests can potentially provide habitat for tree species. 


\section{References}

Altieri MA. 1999. The ecological role of biodiversity in agroecosystems. Agriculture, Ecosystems and Environment 74:19-31.

[BPS] Biro Pusat Statistik. 2014. Kabupaten Buol Dalam Angka 2014. Buol District in figures 2014. Buol, Indonesia: Biro Pusat Statistik.

Dewi S, Khasanah N, Widayati A. 2013. Capacity Strengthening Approach to Vulnerability Assessment (CaSAVA). In: van Noordwijk M, Lusiana B, Leimona B, Dewi S, Wulandari D, eds. 2013. Negotiationsupport toolkit for learning landscapes. Bogor, Indonesia: World Agroforestry Centre (ICRAF) Southeast Asia Regional Program.

Lusiana et al. 2015. Potential and challenges in implementing the co-investment of ecosystem services scheme in Buol District, Indonesia. Working Paper 211. Bogor, Indonesia: World Agroforestry Centre (ICRAF) Southeast Asia Regional Program.

[MEA] Millennium Ecosystem Assessment. 2005. Ecosystems and human well-being: biodiversity synthesis. Washington DC: World Resources Institute.

Saaty TL. 1980. The analytic hierarchy process: planning, priority setting, resource allocation. New York, USA: McGraw-Hill International.

Villamor G, Desrianti F, Akiefnawati R, Amaruzaman S, van Noordwijk M. 2013. Gender influences decisions to change land-use practices in the tropical forest margins of Jambi, Indonesia. Journal of Mitigation and Adaptation Strategies for Global Change 19(6):735-775.

Wijaya C, Rahayu S, Prasetyo A, Dwiyanti E. 2015. Dynamics of land use/cover change and carbon emission in Buol District, Central Sulawesi. Working Paper 214. Bogor, Indonesia: World Agroforestry Centre (ICRAF) Southeast Asia Regional Program. DOI: http://dx.doi.org/10.5716/WP15725.PDF. 


\section{Appendix}

Table A. Tree and other biodiversity utilization by the local community in the upstream cluster during normal and extreme conditions

\begin{tabular}{|c|c|c|}
\hline Land cover & Normal condition & Extreme condition \\
\hline $\begin{array}{l}\text { Disturbed } \\
\text { forest }\end{array}$ & 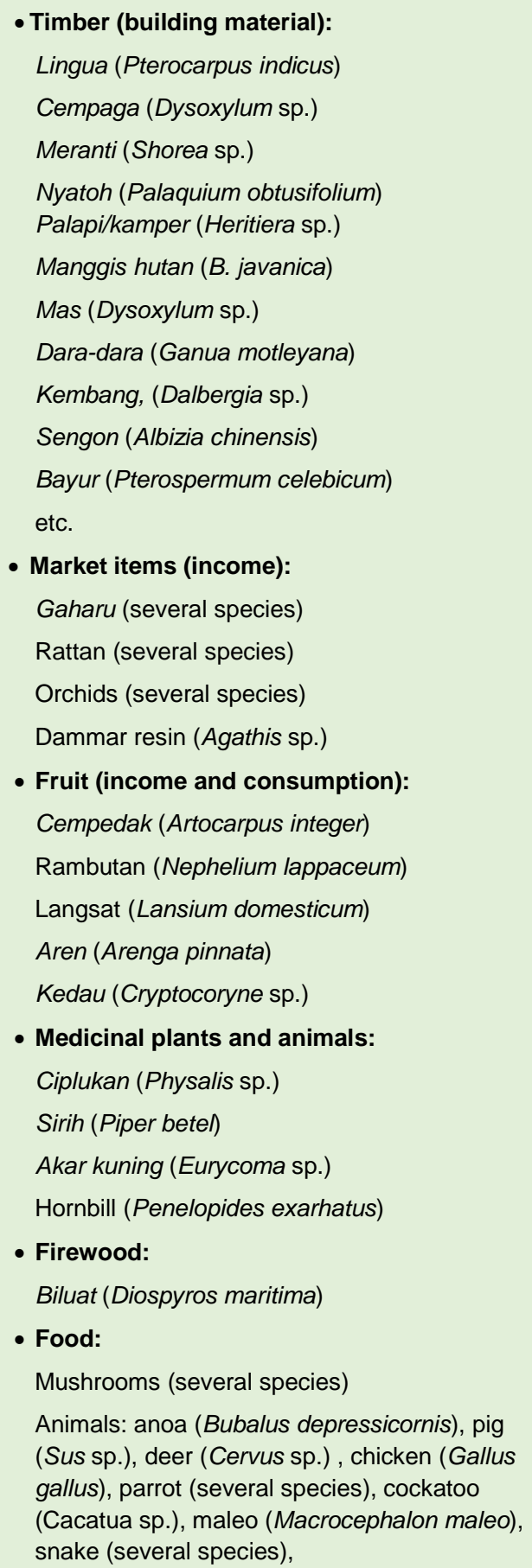 & 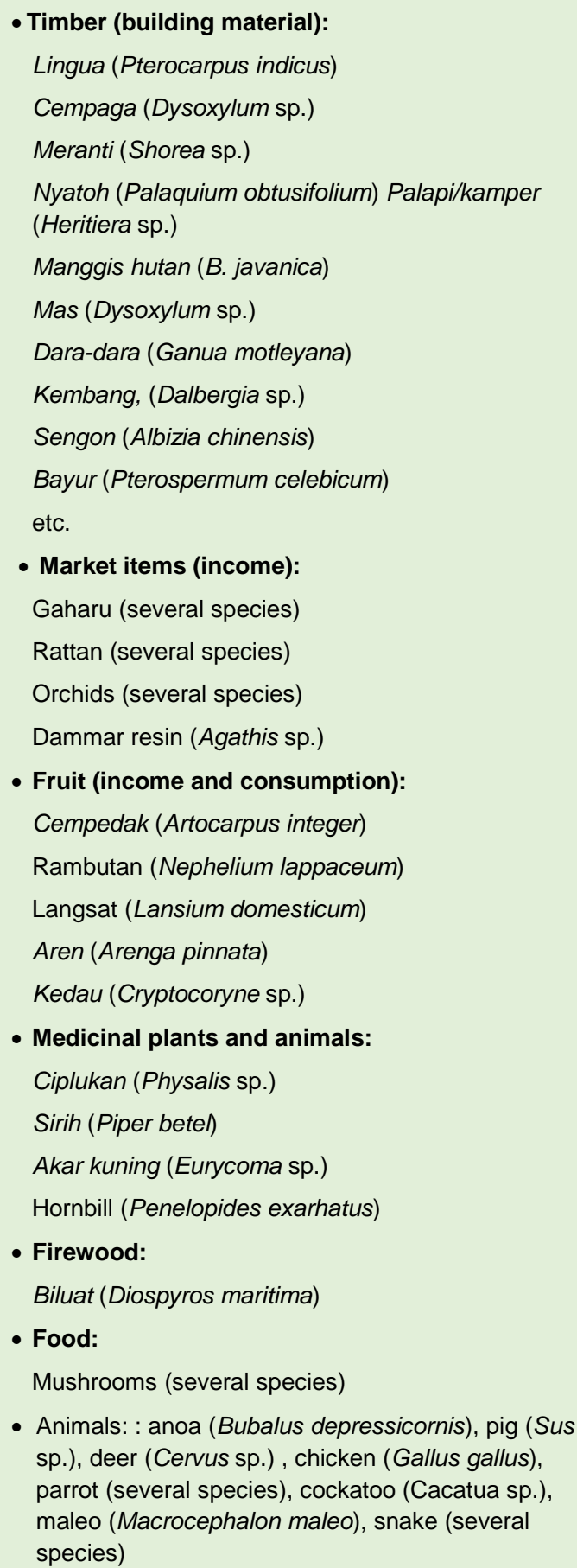 \\
\hline $\begin{array}{l}\text { Oil-palm } \\
\text { plantation }\end{array}$ & $\begin{array}{l}\text { - Market items (income): } \\
\text { Oil palm (Elaeis guineensis) }\end{array}$ & $\begin{array}{l}\text { - Market items (income): } \\
\text { Oil palm (Elaeis guineensis) }\end{array}$ \\
\hline
\end{tabular}




\begin{tabular}{|c|c|c|}
\hline Land cover & Normal condition & Extreme condition \\
\hline & $\begin{array}{l}\text { Others (legume cover crop as fertilizer, } \\
\text { erosion prevention): } \\
\text { Legume (several species: Mucuna sp., } \\
\text { Callopogonium sp.) }\end{array}$ & $\begin{array}{l}\text { - Others (legume cover crop as fertilizer, erosion } \\
\text { prevention): } \\
\text { Legume (several species: Mucuna sp., } \\
\text { Callopogonium sp.) }\end{array}$ \\
\hline Dry land & $\begin{array}{l}\text { - Food and/or market items (income): } \\
\text { Sugarcane (Sacharrum officinarum) } \\
\text { Maize (Zea mays) } \\
\text { Upland rice (Oryza sativa several verieties) } \\
\text { Vegetables (onion, spinach, chilli, kangkung } \\
\text { (Ipomoea aquatica), cabbage, pumpkin, } \\
\text { patchouli, pare (Momordica sp.), lemongrass, } \\
\text { celery, eggplant, cucumber, tomato) } \\
\text { Pulses (string bean, green bean, soybean, } \\
\text { long bean, peanut) } \\
\text { Tubers (for example, gadung (Dioscorea } \\
\text { hispida), ginger, potato, sweet potato) } \\
\text { - Fruits (consumption and income): } \\
\text { Orange (Citrus sp.) } \\
\text { Langsat (Lansium domesticum) } \\
\text { Mango (Mangifera indica) } \\
\text { Coconut (Cocos nucifera) } \\
\text { Rose apple (Syzygium aqueum) } \\
\text { Guava (Psidium guajava) } \\
\text { Kedondong (Spondias dulcis) } \\
\text { Petai (Parkia speciosa) } \\
\text { Jengkol (Archidendron pauciflorum) } \\
\text { Ganemo(Gnetum gnemon) } \\
\text { Snakefruit (Salacca zalacca) } \\
\text { King grass }\end{array}$ & $\begin{array}{l}\text { Food: } \\
\text { Tubers (for example, gadung (Dioscorea hispida), } \\
\text { ginger, potato, sweet potato) }\end{array}$ \\
\hline Rice field & $\begin{array}{l}\text { - Food: } \\
\text { Wet rice (Oryza sativa), } \\
\text { Poultry } \\
\text { Fish (mozambique tilapia (Oreochromis } \\
\text { mossambicus), nile tilapia (Oreochromis } \\
\text { niloticus), catfish (Clarias sp.), carp (Cyprinus } \\
\text { carpio)) } \\
\text { Plants (genjer (Limnocharis flava), kangkung } \\
\text { (Ipomoea aquatica), mendong (Fimbristylis } \\
\text { umbellaris), clover (Marsilea sp.)) }\end{array}$ & $\begin{array}{l}\text { - Food: } \\
\text { Wet rice (Oryza sativa), } \\
\text { Poultry } \\
\text { Fish (mozambique tilapia (Oreochromis } \\
\text { mossambicus), nile tilapia (Oreochromis niloticus), } \\
\text { catfish (Clarias sp.), carp (Cyprinus carpio)) } \\
\text { Plants (genjer (Limnocharis flava), kangkung } \\
\text { (Ipomoea aquatica), mendong (Fimbristylis } \\
\text { umbellaris), clover (Marsilea sp.)) }\end{array}$ \\
\hline $\begin{array}{l}\text { Cacao } \\
\text { agroforest }\end{array}$ & $\begin{array}{l}\text { - Market items (income): } \\
\text { Cacao (Theobroma cacao) } \\
\text { Coffee (Coffea sp.) } \\
\text { - Fruits (consumption and income): } \\
\text { Durian (Durio zibethinus) } \\
\text { Langsat (Lansium domesticum) } \\
\text { Rambutan (Nephelium lappaceum) } \\
\text { Coconut (Cocos nucifera) } \\
\text { Banana (Musa xparadisiaca) }\end{array}$ & $\begin{array}{l}\text { - Market items (income): } \\
\text { Cacao (Theobroma cacao) } \\
\text { Coffee (Coffea sp.) } \\
\text { - Fruits (consumption and income): } \\
\text { Durian (Durio zibethinus) } \\
\text { Langsat (Lansium domesticum) } \\
\text { Rambutan (Nephelium lappaceum) } \\
\text { Coconut (Cocos nucifera) } \\
\text { Banana (Musa xparadisiaca) }\end{array}$ \\
\hline
\end{tabular}




\begin{tabular}{|c|c|c|}
\hline Land cover & Normal condition & Extreme condition \\
\hline & $\begin{array}{l}\text { Petai (Parkia speciosa) } \\
\text { Rose apple (Syzigium aqueum) } \\
\text { Jengkol (Archidendron pauciflorum) } \\
\text { Soursop (Annona muricata) } \\
\text { - Timber (building material): } \\
\text { Cempaga (Dysoxylum sp.) } \\
\text { Teak (Tectona grandis) } \\
\text { Nyatoh (Palaquium obtusifolium) } \\
\text { - Annual crops: } \\
\text { Patchouli (Pogostemon cablin) } \\
\text { Chilli (Capsicum sp.) } \\
\text { - Shade trees: } \\
\text { Gliricidia sepium } \\
\text { Erithryna sp. } \\
\text { - Medicine: } \\
\text { Physalis sp. }\end{array}$ & $\begin{array}{l}\text { Petai (Parkia speciosa) } \\
\text { Rose apple (Syzigium aqueum) } \\
\text { Jengkol (Archidendron pauciflorum) } \\
\text { Soursop (Annona muricata) } \\
\text { - Timber (building material): } \\
\text { Cempaga (Dysoxylum sp.) } \\
\text { Teak (Tectona grandis) } \\
\text { Nyatoh (Palaquium obtusifolium) } \\
\text { - Shade trees: } \\
\text { Gliricidia sepium } \\
\text { Erithryna sp. } \\
\text { - Medicine: } \\
\text { Physalis sp. }\end{array}$ \\
\hline Swamp & $\begin{array}{l}\text { - Food: } \\
\text { Sago (Metroxylon sagu) } \\
\text { - Fencing: } \\
\text { Ketapang (Terminalia catappa) }\end{array}$ & $\begin{array}{l}\text { - Food: } \\
\text { Sago (Metroxylon sagu) } \\
\text { - Fencing: } \\
\text { Ketapang (Terminalia catappa) }\end{array}$ \\
\hline Shrub & $\begin{array}{l}\text { - Timber (building material): } \\
\text { Nyatoh (Palaquium obtusifolium) } \\
\text { Cananga (Cananga odorata) } \\
\text { Jabon (Anthocephalus chinensis) } \\
\text { Gempol (Neonauclea sp.) } \\
\text { - Firewood: } \\
\text { Manggis hutan (Baccaurea javanica) } \\
\text { - Fruits (consumption and income): } \\
\text { Cempedak (Artocarpus integer) } \\
\text { Rambutan (Nephelium lappaceum) } \\
\text { Langsat (Lansium domesticum) } \\
\text { Mango (Mangifera indica) } \\
\text { - Building material (and/or income): } \\
\text { Bamboo } \\
\text { - Animals (food or income): } \\
\text { Snake, chicken, pig, deer }\end{array}$ & $\begin{array}{l}\text { - Timber (building material): } \\
\text { Nyatoh (Palaquium obtusifolium) } \\
\text { Cananga (Cananga odorata) } \\
\text { Jabon (Anthocephalus chinensis) } \\
\text { Gempol (Neonauclea sp.) } \\
\text { - Firewood: } \\
\text { Manggis hutan (Baccaurea javanica) } \\
\text { - Fruits (consumption and income): } \\
\text { Cempedak (Artocarpus integer) } \\
\text { Rambutan (Nephelium lappaceum) } \\
\text { Langsat (Lansium domesticum) } \\
\text { Mango (Mangifera indica) }\end{array}$ \\
\hline
\end{tabular}

* Local name mentioned by the communities during the discussion, need further survey to clarify the species 
Table B. Tree and other biodiversity utilization by the local community in the midstream cluster during normal and extreme conditions

\begin{tabular}{|c|c|c|}
\hline Land cover & Normal condition & Extreme condition \\
\hline $\begin{array}{l}\text { Degraded } \\
\text { forest }\end{array}$ & 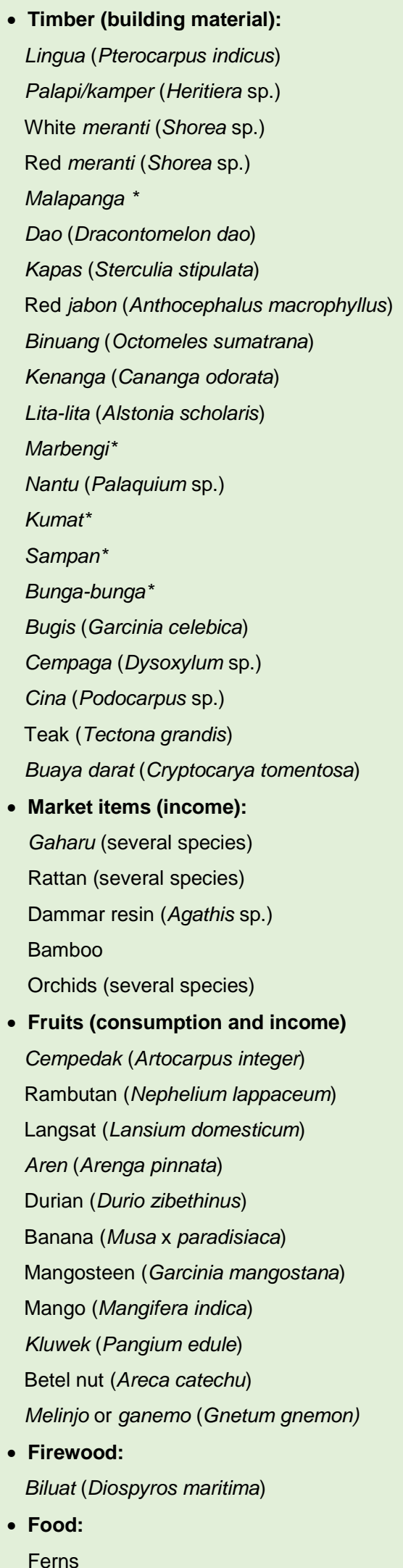 & 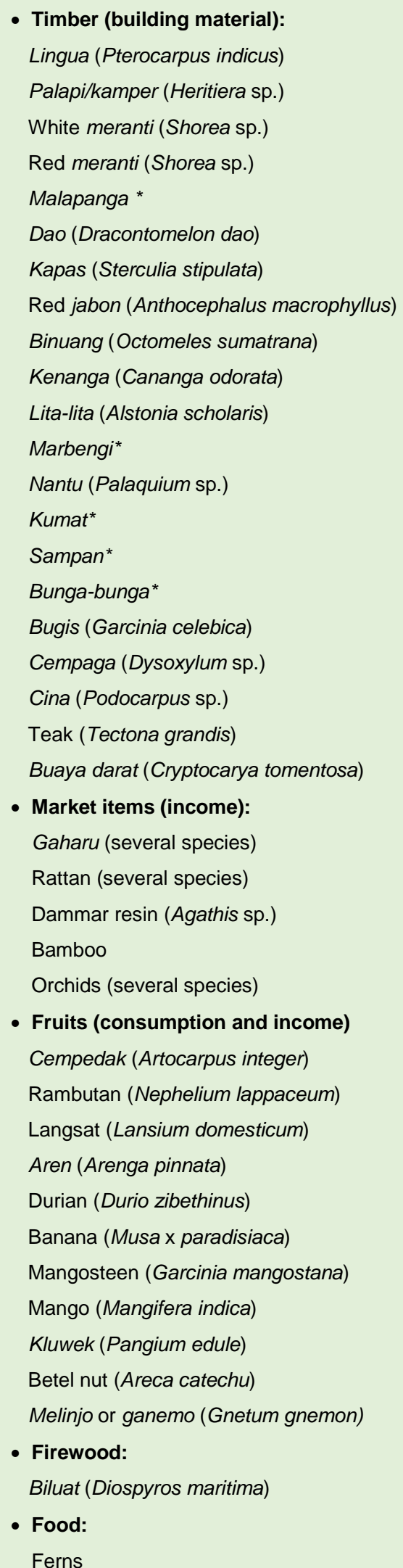 \\
\hline
\end{tabular}




\begin{tabular}{|c|c|c|}
\hline Land cover & Normal condition & Extreme condition \\
\hline & $\begin{array}{l}\text { Honey } \\
\text { Animals (anoa, chicken, hornbill, deer, fish, } \\
\text { pig, snake) }\end{array}$ & $\begin{array}{l}\text { Honey } \\
\text { Animals (anoa, chicken, hornbill, deer, fish, pig, snake) }\end{array}$ \\
\hline $\begin{array}{l}\text { Oil-palm } \\
\text { plantation }\end{array}$ & $\begin{array}{l}\text { - Market items (income): } \\
\text { Oil palm (Elaeis guineensis) }\end{array}$ & $\begin{array}{l}\text { - Market items (income): } \\
\text { Oil palm (Elaeis guineensis) }\end{array}$ \\
\hline Dry land & $\begin{array}{l}\text { - Food: } \\
\text { Maize (Zea mays) } \\
\text { Upland rice (Oryza sativa, several varieties) } \\
\text { Vegetables (onion, spinach, chilli, leek, } \\
\text { cabbage, pumpkin, patchouli, pare } \\
\text { (Momordica sp.), pepper, mustard cabbage, } \\
\text { lemongrass, eggplant, tomato) } \\
\text { Pulses (green bean, soy bean, string bean, } \\
\text { long bean, peanuts) } \\
\text { Tubers (cassava, sweet potato) } \\
\text { Spices (ginger, kencur (Kaempferia } \\
\text { galanga), turmeric) } \\
\text { - Timbers (building material): } \\
\text { Jabon (Anthocephalus chinensis) } \\
\text { Acacia sp. } \\
\text { - Building material: } \\
\text { Bamboo } \\
\text { - Fruits (consumption and income): } \\
\text { Rambutan (Nephelium lappaceum) } \\
\text { Cempedak (Artocarpus integer) } \\
\text { Durian (Durio zibethinus) } \\
\text { Aren (Arenga pinnata) } \\
\text { Coconut (Cocos nucifera) } \\
\text { Langsat (Lansium domesticum) } \\
\text { Cashew (Anacardium occidentale) } \\
\text { Petai (Parkia speciosa) } \\
\text { Jengkol (Archidendron pauciflorum) } \\
\text { Jackfruit (Artocarpus heterophyllus) } \\
\text { Fertilizer (Leucaena sp.) } \\
\text { Others: } \\
\text { The }\end{array}$ & $\begin{array}{l}\text { - Food: } \\
\text { Upland rice (Oryza sativa, several varieties) } \\
\text { - Timbers (building material): } \\
\text { Jabon (Anthocephalus chinensis) } \\
\text { Acacia sp. } \\
\text { - Building material: } \\
\text { Bamboo } \\
\text { - Fruits (consumption and income): } \\
\text { Rambutan (Nephelium lappaceum) } \\
\text { Cempedak (Artocarpus integer) } \\
\text { Durian (Durio zibethinus) } \\
\text { Aren (Arenga pinnata) } \\
\text { Coconut (Cocos nucifera) } \\
\text { Langsat (Lansium domesticum) } \\
\text { Cashew (Anacardium occidentale) } \\
\text { Petai (Parkia speciosa) } \\
\text { Jengkol (Archidendron pauciflorum) } \\
\text { Jackfruit (Artocarpus heterophyllus) } \\
\text { Others: } \\
\text { Fertilizer (Leucaena sp.) }\end{array}$ \\
\hline Rice field & $\begin{array}{l}\text { - Food: } \\
\text { Wet rice (Oryza sativa) } \\
\text { Sago (Metroxylon sagu) } \\
\text { - Vegetables: } \\
\text { Genjer (Limnocharis flava) } \\
\text { Kangkung (Ipomoea aquatica) } \\
\text { - Fish: } \\
\text { Snakehead fish (Channa sp.) } \\
\text { Sepat (Trichogaster trichopterus) } \\
\text { Catfish (Clarias sp.) } \\
\text { Segill* } \\
\text { Mozambique tilapia (Oreochromis } \\
\text { mossambicus) }\end{array}$ & $\begin{array}{l}\text { - Food: } \\
\text { Sago (Metroxylon sagu) }\end{array}$ \\
\hline
\end{tabular}




\begin{tabular}{|c|c|c|}
\hline Land cover & Normal condition & Extreme condition \\
\hline & $\begin{array}{l}\text { - Water birds (as food): } \\
\text { Waterhen (Amaurornis phoenicurus) } \\
\text { Rails* } \\
\text { - Handicraft : } \\
\text { Mendong grass (Fimbristylis umbellaris) }\end{array}$ & \\
\hline $\begin{array}{l}\text { Cacao } \\
\text { agroforest }\end{array}$ & $\begin{array}{l}\text { - Fruits (consumption and income): } \\
\text { Durian (Durio zibethinus) } \\
\text { Langsat (Lansium domesticum) } \\
\text { Rambutan (Nephelium lappaceum) } \\
\text { Coconut (Cocos nucifera) } \\
\text { Banana (Musa x paradisiaca) } \\
\text { Petai (Parkia speciosa) } \\
\text { Rose apple (Syzigium aqueum) } \\
\text { Guava (Psidium guajava) } \\
\text { Mango (Mangifera indica) } \\
\text { Lemon (Citrus sp.) } \\
\text { Snakefruit (Salacca zalacca) } \\
\text { Kapok (Ceiba pentandra) } \\
\text { Papaya (Carica papaya) } \\
\text { Betel nut (Areca catechu) } \\
\text { Timber (building material): } \\
\text { Jabon/kayu mas (Anthocephalus chinensis) } \\
\text { Gmelina (Gmelina arborea) } \\
\text { Market items (income): } \\
\text { Coffee (Coffea spp.) } \\
\text { Cacao (Theobroma cacao) } \\
\text { Clove (Syzygium aromaticum) }\end{array}$ & $\begin{array}{l}\text { - Fruits (consumption and income): } \\
\text { Durian (Durio zibethinus) } \\
\text { Langsat (Lansium domesticum) } \\
\text { Rambutan (Nephelium lappaceum) } \\
\text { Coconut (Cocos nucifera) } \\
\text { Banana (Musa x paradisiaca) } \\
\text { Petai (Parkia speciosa) } \\
\text { Rose apple (Syzigium aqueum) } \\
\text { Guava (Psidium guajava) } \\
\text { Mango (Mangifera indica) } \\
\text { Lemon (Citrus sp.) } \\
\text { Snakefruit (Salacca zalacca) } \\
\text { Kapok (Ceiba pentandra) } \\
\text { Papaya (Carica papaya) } \\
\text { Betel nut (Areca catechu) } \\
\text { Timber (building material): } \\
\text { Jabon/kayu mas (Anthocephalus chinensis) } \\
\text { Gmelina (Gmelina arborea) } \\
\text { Market items (income): } \\
\text { Coffee (Coffea spp.) } \\
\text { Cacao (Theobroma cacao) } \\
\text { Clove (Syzygium aromaticum) }\end{array}$ \\
\hline Swamp & $\begin{array}{l}\text { - Timber (building material): } \\
\text { Ketapang (Terminalia catappa) } \\
\text { Palapi/kamper (Heritiera sp.) } \\
\text { Nantu (Endiandra sp.) } \\
\text { Jabon/kayu mas (Anthocephalus chinensis) } \\
\text { Buaya darat (Cryptocarya tomentosa) } \\
\text { - Food: } \\
\text { Sago (Metroxylon sagu) }\end{array}$ & $\begin{array}{l}\text { - Timber (building material): } \\
\text { Ketapang (Terminalia catappa) } \\
\text { Palapi/kamper (Heritiera sp.) } \\
\text { Nantu (Endiandra sp.) } \\
\text { Jabon/kayu mas (Anthocephalus chinensis) } \\
\text { Buaya darat (Cryptocarya tomentosa) } \\
\text { - Food: } \\
\text { Sago (Metroxylon sagu) }\end{array}$ \\
\hline Shrub & $\begin{array}{l}\text { - Food: } \\
\text { Sago (Metroxylon sagu) } \\
\text { - Fruits (consumption and income): } \\
\text { Durian (Durio zibethinus) } \\
\text { Langsat (Lansium domesticum) } \\
\text { Rambutan (Nephelium lappaceum) } \\
\text { Coconut (Cocos nucifera) } \\
\text { Banana (Musa x paradisiaca) } \\
\text { Petai (Parkia speciosa) } \\
\text { Rose apple (Syzigium aqueum) } \\
\text { Jengkol (Archidendron pauciflorum) }\end{array}$ & $\begin{array}{l}\text { - Food: } \\
\text { Sago (Metroxylon sagu) } \\
\text { - Fruits (consumption and income): } \\
\text { Durian (Durio zibethinus) } \\
\text { Langsat (Lansium domesticum) } \\
\text { Rambutan (Nephelium lappaceum) } \\
\text { Coconut (Cocos nucifera) } \\
\text { Banana (Musa x paradisiaca) } \\
\text { Petai (Parkia speciosa) } \\
\text { Rose apple (Syzigium aqueum) } \\
\text { Jengkol (Archidendron pauciflorum) }\end{array}$ \\
\hline
\end{tabular}




\begin{tabular}{|c|c|c|}
\hline Land cover & Normal condition & Extreme condition \\
\hline & $\begin{array}{l}\text { - Timber (building material): } \\
\text { Teak (Tectona grandis) } \\
\text { Kayu tutup/gempol (Neonauclea sp.) } \\
\text { Jabon (Anthocephalus chinensis) } \\
\text { Sirih (Piper aduncum) } \\
\text { Tinta (Melastoma sp.) } \\
\text { Pulai (Alstonia scholaris) } \\
\text { - Animals (as food): } \\
\text { Water monitor (Varanus salvator) } \\
\text { Waterhen (Amaurornis phoenicurus) } \\
\text { Birds } \\
\text { Rodents } \\
\text { Deer } \\
\text { Wild pig } \\
\text { Building material: } \\
\text { Bamboo } \\
\text { Shade tree: } \\
\text { Gamal (Gliricidia sepium) } \\
\text { Market items (income): } \\
\text { Coffee (Coffea spp.) } \\
\text { Cacao (Theobroma cacao) } \\
\text { Clove (Syzygium aromaticum) } \\
\text { Fodder: } \\
\text { Grass (for example, Eleusine indica) }\end{array}$ & $\begin{array}{l}\text { - Timber (building material): } \\
\text { Teak (Tectona grandis) } \\
\text { Kayu tutup/gempol (Neonauclea sp.) } \\
\text { Jabon (Anthocephalus chinensis) } \\
\text { Sirih (Piper aduncum) } \\
\text { Tinta (Melastoma sp.) } \\
\text { Pulai (Alstonia scholaris) } \\
\text { - Animals (as food): } \\
\text { Water monitor (Varanus salvator) } \\
\text { Waterhen (Amaurornis phoenicurus) } \\
\text { Birds } \\
\text { Rodents } \\
\text { Deer } \\
\text { Wild pig } \\
\text { Building material: } \\
\text { Bamboo } \\
\text { - Shade tree: } \\
\text { Gamal (Gliricidia sepium) } \\
\text { Market items (income): } \\
\text { Coffee (Coffea spp.) } \\
\text { Cacao (Theobroma cacao) } \\
\text { Clove (Syzygium aromaticum) } \\
\text { - Godder: } \\
\text { Grass (for example, Eleusine indica) }\end{array}$ \\
\hline
\end{tabular}

* Local name mentioned by the communities during the discussion, need further survey to clarify the species

Table C. Tree and other biodiversity utilization by the local community in the coastal cluster during normal and extreme conditions

\begin{tabular}{|c|c|c|}
\hline Land cover & Normal condition & Extreme condition \\
\hline $\begin{array}{l}\text { Disturbed } \\
\text { forest }\end{array}$ & $\begin{array}{l}\text { - Timber (building material): } \\
\text { Palapi/kamper (Heritiera sp.) } \\
\text { Meranti (Shorea sp.) } \\
\text { Lingua (Pterocarpus indicus) } \\
\text { Kayu merah* } \\
\text { Ironwood (Instia bijuga) } \\
\text { - } \text { Food: } \\
\text { Sago (Metroxylon sagu) } \\
\text { - Market items (income): } \\
\text { Rattan (several species) } \\
\text { Nibung (Oncosperma sp.) } \\
\text { Dammar } \\
\text { Bamboo } \\
\text { - Fruits: } \\
\text { Durian (Durio zibethinus) } \\
\text { Rambutan (Nephelium lappaceum) } \\
\text { Kapok (Ceiba pentandra) } \\
\text { Aren (Arenga pinnata) } \\
\text { - Animals (as food): } \\
\text { Anoa (Bubalus depressicornis), pig (Sus } \\
\text { sp.), deer (Cervus sp.), chicken (Gallus } \\
\text { gallus), parrot (several species), cockatoo }\end{array}$ & $\begin{array}{l}\text { - Timber (building material): } \\
\text { Palapi/kamper (Heritiera sp.) } \\
\text { Meranti (Shorea sp.) } \\
\text { Lingua (Pterocarpus indicus) } \\
\text { Kayu merah* } \\
\text { Ironwood (Instia bijuga) } \\
\text { - } \text { Food: } \\
\text { Sago (Metroxylon sagu) } \\
\text { - Market items (income): } \\
\text { Rattan (several species) } \\
\text { Nibung (Oncosperma sp.) } \\
\text { Dammar } \\
\text { Bamboo } \\
\text { - Fruits: } \\
\text { Durian (Durio zibethinus) } \\
\text { Rambutan (Nephelium lappaceum) } \\
\text { Kapok (Ceiba pentandra) } \\
\text { Aren (Arenga pinnata) } \\
\text { - Animals (as food): } \\
\text { Anoa (Bubalus depressicornis), pig (Sus sp.), deer } \\
\text { (Cervus sp.), chicken (Gallus gallus), parrot (several } \\
\text { species), cockatoo (Cacatua sp.), maleo }\end{array}$ \\
\hline
\end{tabular}




\begin{tabular}{|c|c|c|}
\hline & $\begin{array}{l}\text { (Cacatua sp.), maleo (Macrocephalon } \\
\text { maleo) } \\
\text { - Animals (as medicine): } \\
\text { Hornbill (Penelopides exarhatus) }\end{array}$ & $\begin{array}{l}\text { (Macrocephalon maleo) } \\
\text { - Animals (as medicine): } \\
\text { Hornbill (Penelopides exarhatus) }\end{array}$ \\
\hline Mangrove & $\begin{array}{l}\text { - Timber (building material): } \\
\text { Sonneratia sp. } \\
\text { Rhizophora sp. } \\
\text { Bruguiera sp. } \\
\text { Mandoti* } \\
\text { Xylocarpus sp. } \\
\text { Nypa fruticans } \\
\text { - Food: } \\
\text { Fish (ikan buronan, ikan tagogo, ikan } \\
\text { bibitan, ikan sako, ikan tabobogug, ikan } \\
\text { tagut)* } \\
\text { Crab, shrimp, sea cucumber, clam, sea eel, } \\
\text { squid, octopus } \\
\text { Birds }\end{array}$ & $\begin{array}{l}\text { Timber (building material): } \\
\text { Sonneratia sp. } \\
\text { Rhizophora sp. } \\
\text { Bruguiera sp. } \\
\text { Mandoti* } \\
\text { Xylocarpus sp. } \\
\text { Nypa fruticans } \\
\text { - Food: } \\
\text { Fish (ikan buronan, ikan tagogo, ikan bibitan, ikan } \\
\text { sako, ikan tabobogug, ikan tagut)* } \\
\text { Crab, shrimp, sea cucumber, clam, sea eel, squid, } \\
\text { octopus } \\
\text { Birds }\end{array}$ \\
\hline $\begin{array}{l}\text { Complex } \\
\text { agroforest }\end{array}$ & $\begin{array}{l}\text { - Timber (building material): } \\
\text { Nyatoh (Palaquium obtusifolium) } \\
\text { Teak (Tectona grandis) } \\
\text { Sengon (Falcataria moluccana) } \\
\text { Rikak* } \\
\text { Yokinak* } \\
\text { Kayu sumbai* } \\
\text { - Fruits: } \\
\text { Coconut (Cocos nucifera) } \\
\text { Nutmeg (Myristica fragrans) } \\
\text { Papaya (Carica papaya) } \\
\text { Langsat (Lansium domesticum) } \\
\text { Durian (Durio zibethinus) } \\
\text { Kedondong (Spondias dulcis) } \\
\text { - Market items (income): } \\
\text { Coffee (Coffea spp.) } \\
\text { Cacao (Theobroma cacao) } \\
\text { Clove (Syzygium aromaticum) } \\
\text { - Food: } \\
\text { Maize (Zea mays) } \\
\text { Cassava (Manihot esculenta) } \\
\text { - Vegetables: } \\
\text { Leek (Allium sp.) } \\
\text { Pepper (Piper sp.) } \\
\text { - Pulses: } \\
\text { Green bean, long bean, peanut } \\
\text { - Medicine: } \\
\text { Turmeric (Curcuma domestica) } \\
\text { Temulawak (Curcuma xanthorizha) } \\
\text { Kumis kucing (Ortosiphon grandiflorus), } \\
\text { Bole bute* } \\
\text { Tonggu yapung* } \\
\text { Putik manuk* } \\
\text { Ramayana (Cassia sp.) } \\
\text { - Airewood: } \\
\text { Kayu sumbai* } \\
\text { Poultry, sheep, cattle, quail, other birds }\end{array}$ & 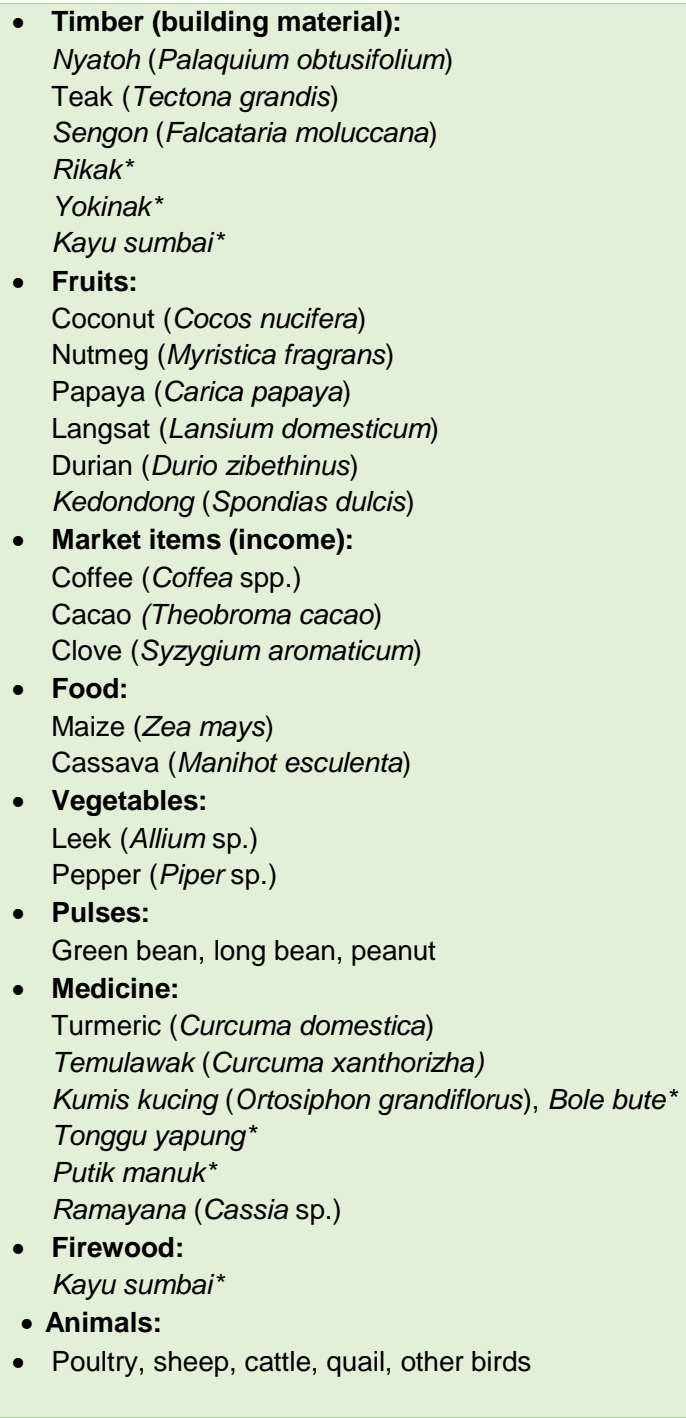 \\
\hline Rice field & $\begin{array}{l}\text { - Food: } \\
\text { Wet rice (Oryza sativa) } \\
\text { - Animals: } \\
\text { Birds } \\
\text { Snakehead fish (Canna sp.) }\end{array}$ & $\begin{array}{l}\text { - Food: } \\
\text { Wet rice (Oryza sativa) } \\
\text { - Animals: } \\
\text { Birds } \\
\text { Snakehead fish (Canna sp.) }\end{array}$ \\
\hline Coconut & $\begin{array}{l}\text { - Market items (income): } \\
\text { Coconut (Cocos nucifera) }\end{array}$ & $\begin{array}{l}\text { - Market items (income): } \\
\text { Coconut (Cocos nucifera) }\end{array}$ \\
\hline
\end{tabular}




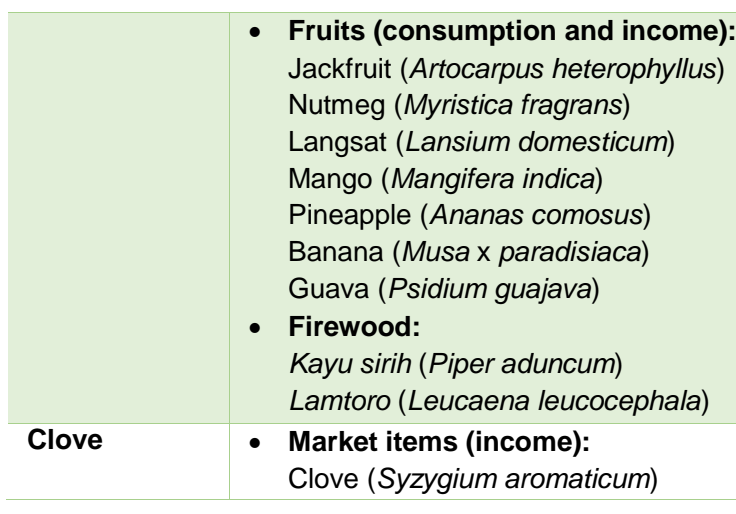

- Fruits (consumption and income): Jackfruit (Artocarpus heterophyllus) Nutmeg (Myristica fragrans) Langsat (Lansium domesticum) Mango (Mangifera indica) Pineapple (Ananas comosus) Banana (Musa x paradisiaca) Guava (Psidium guajava)

- Firewood: Kayu sirih (Piper aduncum)

- Lamtoro (Leucaena leucocephala)

- Market items (income): Clove (Syzygium aromaticum)

* Local name mentioned by the communities during the discussion, need further survey to clarify the species

Table D. List of tree species found on various land-use in Buol district

\begin{tabular}{|c|c|c|c|c|c|}
\hline \multirow{2}{*}{ Land-use } & \multirow{2}{*}{ Tree species } & \multicolumn{4}{|c|}{ Number of individuals at each growth stage } \\
\hline & & Seedling & Sapling & Pole & Tree \\
\hline \multirow[t]{26}{*}{ Cacao agroforest } & Aglaia sp. & & & & 2 \\
\hline & Artocarpus altilis & & & & 1 \\
\hline & Artocarpus heterophyllus & & & & 2 \\
\hline & Artocarpus integer & & & & 1 \\
\hline & Artocarpus lanceifolius & & & & 1 \\
\hline & Cananga odorata & & & & 1 \\
\hline & Ceiba pentandra & & & & 2 \\
\hline & Cocos nucifera & & & & 18 \\
\hline & Coffea canephora & 6 & & & \\
\hline & Dillenia ochreata & & & & 1 \\
\hline & Durio zibethinus & & & 1 & 16 \\
\hline & Ficus septica & & 1 & 3 & \\
\hline & Gliricidia sepium & 6 & & 4 & \\
\hline & Kleinhovia hospita & & & 3 & 3 \\
\hline & Leucaena leucocephala & 1 & 3 & & 4 \\
\hline & Macaranga hispida & 1 & & & \\
\hline & Mangifera indica & & & & 3 \\
\hline & Melastoma malabathricum & & 1 & & \\
\hline & Nephelium lappaceum & 6 & 1 & 1 & 7 \\
\hline & Piper aduncum & & 10 & & \\
\hline & Planchonia valida & & & & 1 \\
\hline & Psidium guajava & & & 1 & \\
\hline & Pycnarrhena cauliflora & & & & 1 \\
\hline & Saurauia tristyla & & 2 & & \\
\hline & Syzygium aqueum & & 4 & & \\
\hline & Syzygium jambos & 3 & & & 1 \\
\hline
\end{tabular}




\begin{tabular}{|c|c|c|c|c|c|}
\hline \multirow{2}{*}{ Land-use } & \multirow{2}{*}{ Tree species } & \multicolumn{4}{|c|}{ Number of individuals at each growth stage } \\
\hline & & Seedling & Sapling & Pole & Tree \\
\hline & Theobroma cacao & & 2 & 13 & 284 \\
\hline & Trema orientalis & & & 1 & \\
\hline & Unidentified 22 & & 4 & & \\
\hline & Villebrunea rubescens & & 1 & 1 & \\
\hline & Vitex quinata & & & & 1 \\
\hline \multirow[t]{16}{*}{ Clove agroforest } & Barringtonia apiculata & 3 & & & \\
\hline & Carica papaya & & & 5 & \\
\hline & Cocos nucifera & & & & 40 \\
\hline & Gliricidia sepium & & 2 & & \\
\hline & Gliricidia sp. & & & & 3 \\
\hline & Mallotus moluccanus & & & & 1 \\
\hline & Musa sp. & & 2 & 2 & \\
\hline & Osmoxylon sp. & & & 4 & \\
\hline & Piper aduncum & & 2 & & \\
\hline & Psidium guajava & 13 & & & 1 \\
\hline & Spondias pinnata & & & 1 & \\
\hline & Syzygium aromaticum & & & & 58 \\
\hline & Tabernaemontana orientalis & 6 & & 1 & \\
\hline & Theobroma cacao & & & 1 & 3 \\
\hline & Unidentified 1 & & & & 1 \\
\hline & Unidentified 6 & & 8 & 1 & \\
\hline \multirow[t]{16}{*}{ Coconut agroforest } & Aglaia sp. & & & 2 & 17 \\
\hline & Alstonia scholaris & 1 & & & \\
\hline & Artocarpus elasticus & & & & 1 \\
\hline & Barringtonia apiculata & & & 1 & \\
\hline & Cananga odorata & 4 & & & \\
\hline & Carica papaya & & & 2 & \\
\hline & Carmona retusa & 1 & & & \\
\hline & Citrus aurantifolia & & & 1 & 1 \\
\hline & Clausena excavata & 2 & & & \\
\hline & Clerodendrum paniculatum & & 1 & & \\
\hline & Cocos nucifera & & 3 & 1 & 124 \\
\hline & Crescentia cujete & 2 & & & \\
\hline & Durio zibethinus & & & & 5 \\
\hline & Elatostema polioneurum & 2 & & & \\
\hline & Ficus septica & & 5 & & \\
\hline & Ficus variegata & & 1 & & \\
\hline
\end{tabular}




\begin{tabular}{|c|c|c|c|c|c|}
\hline \multirow{2}{*}{ Land-use } & \multirow{2}{*}{ Tree species } & \multicolumn{4}{|c|}{ Number of individuals at each growth stage } \\
\hline & & Seedling & Sapling & Pole & Tree \\
\hline & Ganua motleyana & & & & 1 \\
\hline & Gliricidia sepium & & & 2 & 1 \\
\hline & Gliricidia sp. & & & & 8 \\
\hline & Macaranga gigantea & & 2 & & \\
\hline & Mallotus moluccanus & & 3 & & 1 \\
\hline & Mangifera indica & & & & 2 \\
\hline & Melastoma malabathricum & 15 & 4 & & \\
\hline & Metroxylon sagu & & & & 5 \\
\hline & Microcos paniculata & & 4 & & \\
\hline & Musa sp. & & & 1 & \\
\hline & Nephelium lappaceum & & & & 1 \\
\hline & Osmoxylon sp. & & & & 1 \\
\hline & Parinari oblongifolia & & 1 & & \\
\hline & Piper aduncum & & 4 & & 1 \\
\hline & Polyscias nodosa & & & & 1 \\
\hline & Pometia pinnata & & 1 & & \\
\hline & Premna oblongifolia & & 1 & & \\
\hline & Psidium guajava & 14 & 2 & & \\
\hline & Syzygium jambos & 1 & & & \\
\hline & Syzygium malaccense & 1 & & 1 & \\
\hline & Tabernaemontana orientalis & 41 & 2 & & \\
\hline & Tectona grandis & & & & 20 \\
\hline & Theobroma cacao & & 1 & & \\
\hline & Trema orientalis & 1 & & & \\
\hline & Unidentified 1 & & & & 2 \\
\hline & Unidentified 15 & & 1 & & \\
\hline & Unidentified 5 & & & & 3 \\
\hline & Unidentified 7 & & & & 1 \\
\hline Complex agroforest & Acalypha caturus & & & & 8 \\
\hline & Aglaia sp. & 1 & 4 & & 7 \\
\hline & Annona muricata & & & & 1 \\
\hline & Arenga pinnata & & & & 6 \\
\hline & Artocarpus heterophyllus & & & & 10 \\
\hline & Artocarpus lanceifolius & & 10 & 12 & 24 \\
\hline & Averrhoa bilimbi & & & & 2 \\
\hline & Bridelia insulana & & & & 1 \\
\hline & Cananga odorata & & & & 4 \\
\hline
\end{tabular}




\begin{tabular}{|c|c|c|c|c|c|}
\hline \multirow{2}{*}{ Land-use } & \multirow{2}{*}{ Tree species } & \multicolumn{4}{|c|}{ Number of individuals at each growth stage } \\
\hline & & Seedling & Sapling & Pole & Tree \\
\hline & Ceiba pentandra & & & & 7 \\
\hline & Citrus aurantifolia & & & & 4 \\
\hline & Citrus grandis & & & & 1 \\
\hline & Citrus microcarpa & 4 & & & 2 \\
\hline & Citrus sp. & & 2 & 3 & 3 \\
\hline & Citrus $x$ limon & 1 & & & \\
\hline & Cocos nucifera & & 1 & & 76 \\
\hline & Codiaeum variegatum & & 2 & & \\
\hline & Crescentia cujete & & 2 & & 4 \\
\hline & Dillenia ochreata & & & 2 & \\
\hline & Dracontomelon dao & & & & 1 \\
\hline & Durio zibethinus & 1 & 6 & 2 & 5 \\
\hline & Dysoxylum parasiticum & 4 & & & \\
\hline & Elaeis guineensis & & 1 & & \\
\hline & Euodia latifolia & 2 & & & \\
\hline & Evodia latifolia & & 1 & 1 & \\
\hline & Ficus septica & & 4 & 1 & 6 \\
\hline & Ficus variegata & & & & 1 \\
\hline & Flacourtia rukam & 2 & 1 & & 11 \\
\hline & Ganua motleyana & & & & 1 \\
\hline & Gliricidia sepium & & 1 & & 4 \\
\hline & Glochidion philippicum & & 1 & & 1 \\
\hline & Jatropha curcas & & & 2 & \\
\hline & Kleinhovia hospita & & & & 7 \\
\hline & Leea aequata & & 1 & & \\
\hline & Litsea mappacea & & 1 & 1 & 12 \\
\hline & Macaranga gigantea & & & & 1 \\
\hline & Mallotus floribundus & & 1 & & \\
\hline & Mangifera indica & & 2 & 2 & 6 \\
\hline & Melastoma malabathricum & 3 & & & \\
\hline & Musa sp. & & 1 & 2 & \\
\hline & Nephelium lappaceum & 2 & 1 & 1 & 11 \\
\hline & Osmoxylon sp. & & & & 1 \\
\hline & Piper aduncum & & 4 & 1 & \\
\hline & Piper betle & & 1 & & \\
\hline & Polyscias nodosa & & & & 2 \\
\hline & Premna foetida & & & & 1 \\
\hline
\end{tabular}




\begin{tabular}{|c|c|c|c|c|c|}
\hline \multirow{2}{*}{ Land-use } & \multirow{2}{*}{ Tree species } & \multicolumn{4}{|c|}{ Number of individuals at each growth stage } \\
\hline & & Seedling & Sapling & Pole & Tree \\
\hline & Psidium guajava & 7 & 1 & & \\
\hline & Spondias dulcis & & & & 1 \\
\hline & Sterculia stipulata & & & 2 & 2 \\
\hline & Syzygium aqueum & 1 & & & \\
\hline & Syzygium malaccense & 1 & 1 & & \\
\hline & Tabernaemontana orientalis & 1 & & & \\
\hline & Terminalia bellirica & 1 & & & \\
\hline & Theobroma cacao & & & 20 & 152 \\
\hline & Unidentified 641 & & & 1 & \\
\hline \multirow[t]{28}{*}{ Disturbed forest } & Aglaia argentea & & & & 4 \\
\hline & Aglaia edulis & 1 & 2 & 1 & 7 \\
\hline & Aglaia malaccensis & & & 1 & \\
\hline & Aglaia odoratissima & & 1 & & 1 \\
\hline & Artocarpus elasticus & 1 & & & \\
\hline & Artocarpus reticulatus & & 3 & 1 & \\
\hline & Atuna excelsa & & 1 & & \\
\hline & Baccaurea javanica & & & & 3 \\
\hline & Cantleya corniculata & 1 & & & \\
\hline & Carallia brachiata & 2 & & & \\
\hline & Crypteronia paniculata & & 1 & & \\
\hline & Cryptocarya cf. tomentosa & & & & 1 \\
\hline & Cynometra ramiflora & & & & 1 \\
\hline & Cyrtandra sp. & 1 & & & \\
\hline & Dillenia ochreata & 2 & 2 & 2 & 8 \\
\hline & Diospyros sp. & & & & 1 \\
\hline & Diospyros species 1 & & & & 1 \\
\hline & Dracontomelon dao & 13 & 7 & 4 & 6 \\
\hline & Dysoxylum sp. & & & & 4 \\
\hline & Elatostema polioneurum & 12 & 1 & & \\
\hline & Elattostachys verrucosa & & 1 & & \\
\hline & Endiandra sp. & & & & 3 \\
\hline & Ficus benjamina & 1 & & & \\
\hline & Ficus lepicarpa & & & & 2 \\
\hline & Ficus septica & & & & 3 \\
\hline & Ganua motleyana & 1 & 2 & 1 & 3 \\
\hline & Garcinia celebica & 1 & & & \\
\hline & Gnetum latifolium & & & & 3 \\
\hline
\end{tabular}




\begin{tabular}{|c|c|c|c|c|c|}
\hline \multirow{2}{*}{ Land-use } & \multirow{2}{*}{ Tree species } & \multicolumn{4}{|c|}{ Number of individuals at each growth stage } \\
\hline & & Seedling & Sapling & Pole & Tree \\
\hline & Gonystylus macrophyllus & & 1 & & \\
\hline & Gyrinops versteegii & & & & 1 \\
\hline & Harpullia arborea & & & 1 & \\
\hline & Ixora species 1 & & & & 2 \\
\hline & Lepiniopsis ternatensis & 1 & 1 & & 1 \\
\hline & Licuala spinosa & & 1 & & \\
\hline & Litsea mappacea & & & & 2 \\
\hline & Macaranga gigantea & & 1 & 3 & 9 \\
\hline & Mallotus floribundus & & 4 & 3 & 2 \\
\hline & Neonauclea calycina & & 1 & & \\
\hline & Nephelium lappaceum & 2 & 2 & 2 & 3 \\
\hline & Octomeles sumatrana & & & & 1 \\
\hline & Osmoxylon sp. & & & 1 & \\
\hline & Palaquium obovatum & & & & 2 \\
\hline & Phaleria cf. capitata & & & 1 & \\
\hline & Piper aduncum & 1 & & & \\
\hline & Planchonia valida & 2 & & & \\
\hline & Polyalthia lateriflora & 4 & 1 & 2 & 3 \\
\hline & Pterocarpus indicus & 1 & & & 1 \\
\hline & Pterospermum celebicum & & & & 1 \\
\hline & Saurauia tristyla & & 1 & & \\
\hline & Schima wallichii & & 1 & & \\
\hline & Shorea leprosula & & & & 1 \\
\hline & Shorea parvifolia & & & & 1 \\
\hline & Spathiostemon javensis & & 4 & & \\
\hline & Terminalia bellirica & & & & 2 \\
\hline & Uncaria cf. gambir & & 1 & & \\
\hline & Unidentified 10 & & & 1 & 2 \\
\hline & Unidentified 12 & & & & 1 \\
\hline & Unidentified 13 & & & & 1 \\
\hline & Unidentified 14 & & & & 2 \\
\hline & Unidentified 16 & 1 & 4 & & \\
\hline & Unidentified 17 & & 1 & & \\
\hline & Unidentified 18 & & 2 & & \\
\hline & Unidentified 19 & & & & 1 \\
\hline & Unidentified 20 & & & 2 & \\
\hline & Unidentified 21 & & & 1 & \\
\hline
\end{tabular}




\begin{tabular}{|c|c|c|c|c|c|}
\hline \multirow{2}{*}{ Land-use } & \multirow{2}{*}{ Tree species } & \multicolumn{4}{|c|}{ Number of individuals at each growth stage } \\
\hline & & Seedling & Sapling & Pole & Tree \\
\hline & Unidentified 23 & & 2 & & \\
\hline & Unidentified 24 & 1 & 1 & & \\
\hline & Unidentified 25 & & 1 & 1 & 6 \\
\hline & Unidentified 26 & & & & 1 \\
\hline & Unidentified 3 & & & & 1 \\
\hline & Unidentified 4 & & & & 1 \\
\hline & Unidentified 8 & & & & 3 \\
\hline & Unidentified 9 & & & & 1 \\
\hline \multirow[t]{18}{*}{ Sago and shrub } & Artocarpus lanceifolius & & & & 1 \\
\hline & Barringtonia apiculata & 11 & & & \\
\hline & Crescentia cujete & & 1 & 1 & \\
\hline & Dillenia ochreata & & & & 1 \\
\hline & Ficus obscura & & 1 & & \\
\hline & Ficus septica & 2 & 2 & & \\
\hline & Ficus variegata & & & & 1 \\
\hline & Glochidion macrocarpum & 7 & & & \\
\hline & Kleinhovia hospita & 6 & 1 & & 6 \\
\hline & Leea angulata & & 1 & & \\
\hline & Metroxylon sagu & & & & 396 \\
\hline & Microcos paniculata & & & & 1 \\
\hline & Morinda sp. & 5 & & & \\
\hline & Neonauclea calycina & & 1 & & \\
\hline & Saurauia tristyla & 2 & & & \\
\hline & Spatholobus littoralis & 1 & 1 & & \\
\hline & Sterculia stipulata & & 1 & 1 & \\
\hline & Unidentified 7 & 1 & & & 5 \\
\hline \multirow[t]{11}{*}{ Teak monoculture } & Averrhoa bilimbi & & & 1 & 1 \\
\hline & Barringtonia apiculata & 2 & 2 & 2 & \\
\hline & Ceiba pentandra & & & & 1 \\
\hline & Durio zibethinus & 1 & & & 1 \\
\hline & Ficus septica & & & 1 & \\
\hline & Flacourtia rukam & & & & 1 \\
\hline & Gliricidia sepium & & & & 2 \\
\hline & Glochidion philippicum & & & & 1 \\
\hline & Leea aequata & & 1 & & \\
\hline & Macaranga gigantea & & & & 6 \\
\hline & Mangifera indica & 1 & & 1 & 1 \\
\hline
\end{tabular}




\begin{tabular}{|c|c|c|c|c|c|}
\hline \multirow{2}{*}{ Land-use } & \multirow{2}{*}{ Tree species } & \multicolumn{4}{|c|}{ Number of individuals at each growth stage } \\
\hline & & Seedling & Sapling & Pole & Tree \\
\hline & Microcos paniculata & & & 1 & \\
\hline & Nephelium lappaceum & 2 & & & \\
\hline & Piper aduncum & & 10 & 1 & \\
\hline & Psidium guajava & 1 & & & \\
\hline & Saurauia tristyla & & 1 & & \\
\hline & Tectona grandis & 3 & 9 & 6 & 269 \\
\hline & Theobroma cacao & & & 1 & 16 \\
\hline & Unidentified 16 & & 1 & & \\
\hline & Unidentified 608 & 1 & 1 & & \\
\hline & Unidentified 643 & & 1 & & \\
\hline \multirow[t]{27}{*}{ Undisturbed forest } & Aglaia argentea & & & & 12 \\
\hline & Aglaia leucophylla & 1 & 4 & 1 & 2 \\
\hline & Aglaia odoratissima & 1 & 1 & 1 & 1 \\
\hline & Anthocephalus chinensis & & & & 1 \\
\hline & Artocarpus elasticus & & & & 3 \\
\hline & Atuna excelsa & & & & 1 \\
\hline & Barringtonia apiculata & 1 & & & \\
\hline & Bombax ceiba & & & & 2 \\
\hline & Bridelia insulana & & & 1 & \\
\hline & Calophyllum soulatri & 5 & 3 & 1 & \\
\hline & Calophyllum species 1 & 1 & 2 & & 2 \\
\hline & Cananga odorata & & 2 & & 5 \\
\hline & Carallia brachiata & & 1 & & 3 \\
\hline & Celtis philippensis & & 2 & & \\
\hline & Crypteronia griffithii & & & & 8 \\
\hline & Cyrtandra sp. & & & & 1 \\
\hline & Dimocarpus longan & 1 & & & \\
\hline & Diospyros cf. maritima & & & & 4 \\
\hline & Diospyros maritima & 5 & 1 & 4 & 17 \\
\hline & Diospyros sp. & 1 & & & \\
\hline & Diospyros species 1 & & & & 2 \\
\hline & Dracontomelon dao & 1 & 1 & 1 & 1 \\
\hline & Durio carinatus & 1 & & & \\
\hline & Evodia latifolia & 2 & & & 3 \\
\hline & Ficus fistulosa & & 1 & & \\
\hline & Ficus sp. & & & 1 & \\
\hline & Ficus subulata & & & & 2 \\
\hline
\end{tabular}




\begin{tabular}{|c|c|c|c|c|c|}
\hline \multirow{2}{*}{ Land-use } & \multirow{2}{*}{ Tree species } & \multicolumn{4}{|c|}{ Number of individuals at each growth stage } \\
\hline & & Seedling & Sapling & Pole & Tree \\
\hline & Ganua motleyana & 1 & & & \\
\hline & Heritiera javanica & 1 & & & \\
\hline & Heritiera littoralis & & & & 1 \\
\hline & Hymenodictyon horsfieldii & & 1 & & 1 \\
\hline & Kleinhovia hospita & & & & 2 \\
\hline & Leea aequata & & & 1 & \\
\hline & Leea angulata & & & & 2 \\
\hline & Licuala spinosa & & 1 & & 38 \\
\hline & Macaranga gigantea & & 1 & & 1 \\
\hline & Macaranga tanarius & & & & 1 \\
\hline & Maesa cf. latifolia & & & 1 & \\
\hline & Mallotus floribundus & & 1 & & \\
\hline & Mangifera laurina & & & & 2 \\
\hline & Octomeles sumatrana & & & & 1 \\
\hline & Palaquium obtusifolium & & & & 1 \\
\hline & Planchonella cf. nitida & 1 & & & \\
\hline & Planchonella nitida & 1 & 1 & & \\
\hline & Plectronia sp. & 2 & & & \\
\hline & Dracaena angustifolia & & 3 & 1 & 2 \\
\hline & Polyalthia lateriflora & 2 & 1 & & 3 \\
\hline & Prainea limpato & 1 & & & \\
\hline & Pterocarpus indicus & & & 1 & \\
\hline & Salacia oblongifolia & 5 & & & \\
\hline & Santiria oblongifolia & & 2 & 1 & 1 \\
\hline & Shorea acuminata & 3 & & & \\
\hline & Shorea parvifolia & & & & 2 \\
\hline & Spathiostemon javensis & 3 & 10 & 3 & 27 \\
\hline & Spondias pinnata & & & & 1 \\
\hline & Sterculia oblongata & 1 & & 1 & \\
\hline & Sterculia stipulata & & & 1 & 2 \\
\hline & Strichnos sp. & 1 & & & 1 \\
\hline & Syzygium malaccense & & & & 1 \\
\hline & Syzygium species 1 & & & & 1 \\
\hline & Unidentified 11 & & & 1 & \\
\hline & Unidentified 24 & & & & 1 \\
\hline & Unidentified 607 & & 1 & & \\
\hline & Unidentified 609 & & & & 1 \\
\hline
\end{tabular}




\begin{tabular}{|c|c|c|c|c|c|}
\hline \multirow{2}{*}{ Land-use } & \multirow{2}{*}{ Tree species } & \multicolumn{4}{|c|}{ Number of individuals at each growth stage } \\
\hline & & Seedling & Sapling & Pole & Tree \\
\hline & Unidentified 610 & 1 & 8 & & 7 \\
\hline & Unidentified 614 & & & & 1 \\
\hline & Unidentified 621 & & & 3 & 1 \\
\hline & Unidentified 624 & & & & 1 \\
\hline & Unidentified 625 & & & 1 & \\
\hline & Unidentified 628 & & & & 1 \\
\hline & Unidentified 642 & 1 & & & \\
\hline & Unidentified 644 & & & & 1 \\
\hline & Unidentified 645 & & & & 1 \\
\hline & Unidentified 646 & & & & 1 \\
\hline & Unidentified 651 & & & & 1 \\
\hline & Xylopia malayana & & & 1 & \\
\hline & Ziziphus javanensis & 1 & & & \\
\hline
\end{tabular}




\section{WORKING PAPERS WITH DOIS}

\section{5}

1. Agroforestry in the drylands of eastern Africa: a call to action

2. Biodiversity conservation through agroforestry: managing tree species diversity within a network of community-based, nongovernmental, governmental and research organizations in western Kenya.

3. Invasion of prosopis juliflora and local livelihoods: Case study from the Lake Baringo area of Kenya

4. Leadership for change in farmers organizations: Training report: Ridar Hotel, Kampala, 29th March to 2nd April 2005.

5. Domestication des espèces agroforestières au Sahel : situation actuelle et perspectives

6. Relevé des données de biodiversité ligneuse: Manuel du projet biodiversité des parcs agroforestiers au Sahel

7. Improved land management in the Lake Victoria Basin: TransVic Project's draft report.

8. Livelihood capital, strategies and outcomes in the Taita hills of Kenya

9. Les espèces ligneuses et leurs usages: Les préférences des paysans dans le Cercle de Ségou, au Mali

10. La biodiversité des espèces ligneuses: Diversité arborée et unités de gestion du terroir dans le Cercle de Ségou, au Mali

\section{6}

11. Bird diversity and land use on the slopes of Mt. Kilimanjaro and the adjacent plains, Tanzania

12. Water, women and local social organization in the Western Kenya Highlands

13. Highlights of ongoing research of the World Agroforestry Centre in Indonesia

14. Prospects of adoption of tree-based systems in a rural landscape and its likely impacts on carbon stocks and farmers' welfare: The FALLOW Model Application in Muara Sungkai, Lampung, Sumatra, in a 'Clean Development Mechanism' context

15. Equipping integrated natural resource managers for healthy Agroforestry landscapes.

17. Agro-biodiversity and CGIAR tree and forest science: approaches and examples from Sumatra.

18. Improving land management in eastern and southern Africa: A review of policies.

19. Farm and household economic study of Kecamatan Nanggung, Kabupaten Bogor, Indonesia: A socioeconomic base line study of Agroforestry innovations and livelihood enhancement.

20. Lessons from eastern Africa's unsustainable charcoal business.

21. Evolution of RELMA's approaches to land management: Lessons from two decades of research and development in eastern and southern Africa

22. Participatory watershed management: Lessons from RELMA's work with farmers in eastern Africa.

23. Strengthening farmers' organizations: The experience of RELMA and ULAMP.

24. Promoting rainwater harvesting in eastern and southern Africa.

25. The role of livestock in integrated land management.

26. Status of carbon sequestration projects in Africa: Potential benefits and challenges to scaling up.

27. Social and Environmental Trade-Offs in Tree Species Selection: A Methodology for Identifying Niche Incompatibilities in Agroforestry [Appears as AHI Working Paper no. 9]

28. Managing tradeoffs in agroforestry: From conflict to collaboration in natural resource management. [Appears as AHI Working Paper no. 10]

29. Essai d'analyse de la prise en compte des systemes agroforestiers pa les legislations forestieres au Sahel: Cas du Burkina Faso, du Mali, du Niger et du Senegal.

30. Etat de la recherche agroforestière au Rwanda etude bibliographique, période 1987-2003 
31. Science and technological innovations for improving soil fertility and management in Africa: A report for NEPAD's Science and Technology Forum.

32. Compensation and rewards for environmental services.

33. Latin American regional workshop report compensation.

34. Asia regional workshop on compensation ecosystem services.

35. Report of African regional workshop on compensation ecosystem services.

36. Exploring the inter-linkages among and between compensation and rewards for ecosystem services CRES and human well-being

37. Criteria and indicators for environmental service compensation and reward mechanisms: realistic, voluntary, conditional and pro-poor

38. The conditions for effective mechanisms of compensation and rewards for environmental services.

39. Organization and governance for fostering Pro-Poor Compensation for Environmental Services.

40. How important are different types of compensation and reward mechanisms shaping poverty and ecosystem services across Africa, Asia \& Latin America over the Next two decades?

41. Risk mitigation in contract farming: The case of poultry, cotton, woodfuel and cereals in East Africa.

42. The RELMA savings and credit experiences: Sowing the seed of sustainability

43. Yatich J., Policy and institutional context for NRM in Kenya: Challenges and opportunities for Landcare.

44. Nina-Nina Adoung Nasional di So! Field test of rapid land tenure assessment (RATA) in the Batang Toru Watershed, North Sumatera.

45. Is Hutan Tanaman Rakyat a new paradigm in community based tree planting in Indonesia?

46. Socio-Economic aspects of brackish water aquaculture (Tambak) production in Nanggroe Aceh Darrusalam.

47. Farmer livelihoods in the humid forest and moist savannah zones of Cameroon.

48. Domestication, genre et vulnérabilité : Participation des femmes, des Jeunes et des catégories les plus pauvres à la domestication des arbres agroforestiers au Cameroun.

49. Land tenure and management in the districts around Mt Elgon: An assessment presented to the Mt Elgon ecosystem conservation programme.

50. The production and marketing of leaf meal from fodder shrubs in Tanga, Tanzania: A pro-poor enterprise for improving livestock productivity.

51. Buyers Perspective on Environmental Services (ES) and Commoditization as an approach to liberate ES markets in the Philippines.

52. Towards Towards community-driven conservation in southwest China: Reconciling state and local perceptions.

53. Biofuels in China: An Analysis of the Opportunities and Challenges of Jatropha curcas in Southwest China.

54. Jatropha curcas biodiesel production in Kenya: Economics and potential value chain development for smallholder farmers

55. Livelihoods and Forest Resources in Aceh and Nias for a Sustainable Forest Resource Management and Economic Progress

56. Agroforestry on the interface of Orangutan Conservation and Sustainable Livelihoods in Batang Toru, North Sumatra. 
57. Assessing Hydrological Situation of Kapuas Hulu Basin, Kapuas Hulu Regency, West Kalimantan.

58. Assessing the Hydrological Situation of Talau Watershed, Belu Regency, East Nusa Tenggara.

59. Kajian Kondisi Hidrologis DAS Talau, Kabupaten Belu, Nusa Tenggara Timur.

60. Kajian Kondisi Hidrologis DAS Kapuas Hulu, Kabupaten Kapuas Hulu, Kalimantan Barat.

61. Lessons learned from community capacity building activities to support agroforest as sustainable economic alternatives in Batang Toru orang utan habitat conservation program (Martini, Endri et al.)

62. Mainstreaming Climate Change in the Philippines.

63. A Conjoint Analysis of Farmer Preferences for Community Forestry Contracts in the Sumber Jaya Watershed, Indonesia.

64. The highlands: a shared water tower in a changing climate and changing Asia

65. Eco-Certification: Can It Deliver Conservation and Development in the Tropics.

66. Designing ecological and biodiversity sampling strategies. Towards mainstreaming climate change in grassland management.

67. Towards mainstreaming climate change in grassland management policies and practices on the Tibetan Plateau

68. An Assessment of the Potential for Carbon Finance in Rangelands

69 ECA Trade-offs Among Ecosystem Services in the Lake Victoria Basin.

69. The last remnants of mega biodiversity in West Java and Banten: an in-depth exploration of RaTA (Rapid Land Tenure Assessment) in Mount Halimun-Salak National Park Indonesia

70. Le business plan d'une petite entreprise rurale de production et de commercialisation des plants des arbres locaux. Cas de quatre pépinières rurales au Cameroun.

71. Les unités de transformation des produits forestiers non ligneux alimentaires au Cameroun. Diagnostic technique et stratégie de développement Honoré Tabuna et Ingratia Kayitavu.

72. Les exportateurs camerounais de safou (Dacryodes edulis) sur le marché sous régional et international. Profil, fonctionnement et stratégies de développement.

73. Impact of the Southeast Asian Network for Agroforestry Education (SEANAFE) on agroforestry education capacity.

74. Setting landscape conservation targets and promoting them through compatible land use in the Philippines.

75. Review of methods for researching multistrata systems.

76. Study on economical viability of Jatropha curcas L. plantations in Northern Tanzania assessing farmers' prospects via cost-benefit analysis

77. Cooperation in Agroforestry between Ministry of Forestry of Indonesia and International Center for Research in Agroforestry

78. "China's bioenergy future. an analysis through the Lens if Yunnan Province

79. Land tenure and agricultural productivity in Africa: A comparative analysis of the economics literature and recent policy strategies and reforms

80. Boundary organizations, objects and agents: linking knowledge with action in Agroforestry watersheds

81. Reducing emissions from deforestation and forest degradation (REDD) in Indonesia: options and challenges for fair and efficient payment distribution mechanisms 
82. Mainstreaming climate change into agricultural education: challenges and perspectives

83. Challenging conventional mindsets and disconnects in conservation: the emerging role of ecoagriculture in Kenya's landscape mosaics

84. Lesson learned RATA garut dan bengkunat: suatu upaya membedah kebijakan pelepasan kawasan hutan dan redistribusi tanah bekas kawasan hutan

85. The emergence of forest land redistribution in Indonesia

86. Commercial opportunities for fruit in Malawi

87. Status of fruit production processing and marketing in Malawi

88. Fraud in tree science

89. Trees on farm: analysis of global extent and geographical patterns of agroforestry

90. The springs of Nyando: water, social organization and livelihoods in Western Kenya

91. Building capacity toward region-wide curriculum and teaching materials development in agroforestry education in Southeast Asia

92. Overview of biomass energy technology in rural Yunnan (Chinese - English abstract)

93. A pro-growth pathway for reducing net GHG emissions in China

94. Analysis of local livelihoods from past to present in the central Kalimantan Ex-Mega Rice Project area

95. Constraints and options to enhancing production of high quality feeds in dairy production in Kenya, Uganda and Rwanda

2010

96. Agroforestry education in the Philippines: status report from the Southeast Asian Network for Agroforestry Education (SEANAFE)

97. Economic viability of Jatropha curcas L. plantations in Northern Tanzania- assessing farmers' prospects via cost-benefit analysis.

98. Hot spot of emission and confusion: land tenure insecurity, contested policies and competing claims in the central Kalimantan Ex-Mega Rice Project area

99. Agroforestry competences and human resources needs in the Philippines

100. CES/COS/CIS paradigms for compensation and rewards to enhance environmental Services

101. Case study approach to region-wide curriculum and teaching materials development in agroforestry education in Southeast Asia

102. Stewardship agreement to reduce emissions from deforestation and degradation (REDD): Lubuk Beringin's Hutan Desa as the first village forest in Indonesia

103. Landscape dynamics over time and space from ecological perspective

104. Komoditisasi atau koinvestasi jasa lingkungan: skema imbal jasa lingkungan program peduli sungai di DAS Way Besai, Lampung, Indonesia

105. Improving smallholders' rubber quality in Lubuk Beringin, Bungo district, Jambi province, Indonesia: an initial analysis of the financial and social benefits

106. Rapid Carbon Stock Appraisal (RACSA) in Kalahan, Nueva Vizcaya, Philippines

107. Tree domestication by ICRAF and partners in the Peruvian Amazon: lessons learned and future prospects in the domain of the Amazon Initiative eco-regional program

108. Memorias del Taller Nacional: "Iniciativas para Reducir la Deforestación en la region Andino Amazónica", 09 de Abril del 2010. Proyecto REALU Peru

109. Percepciones sobre la Equidad y Eficiencia en la cadena de valor de REDD en Perú -Reporte de Talleres en Ucayali, San Martín y Loreto, 2009. Proyecto REALU-Perú. 
110. Reducción de emisiones de todos los Usos del Suelo. Reporte del Proyecto REALU Perú Fase 1

111. Programa Alternativas a la Tumba-y-Quema (ASB) en el Perú. Informe Resumen y Síntesis de la Fase II. 2da. versión revisada

112. Estudio de las cadenas de abastecimiento de germoplasma forestal en la amazonía Boliviana

113. Biodiesel in the Amazon

114. Estudio de mercado de semillas forestales en la amazonía Colombiana

115. Estudio de las cadenas de abastecimiento de germoplasma forestal en Ecuador http://dx.doi.org10.5716/WP10340.PDF

116. How can systems thinking, social capital and social network analysis help programs achieve impact at scale?

117. Energy policies, forests and local communities in the Ucayali Region, Peruvian Amazon

118. NTFPs as a Source of Livelihood Diversification for Local Communities in the Batang Toru Orangutan Conservation Program

119. Studi Biodiversitas: Apakah agroforestry mampu mengkonservasi keanekaragaman hayati di DAS Konto?

120. Estimasi Karbon Tersimpan di Lahan-lahan Pertanian di DAS Konto, Jawa Timur

121. Implementasi Kaji Cepat Hidrologi (RHA) di Hulu DAS Brantas, Jawa Timur. http://dx.doi.org/10.5716/WP10338.PDF

122. Kaji Cepat Hidrologi di Daerah Aliran Sungai Krueng Peusangan, NAD,Sumatra http://dx.doi.org/10.5716/WP10337.PDF

123. A Study of Rapid Hydrological Appraisal in the Krueng Peusangan Watershed, NAD, Sumatra. http://dx.doi.org/10.5716/WP10339.PDF

2011

124. An Assessment of farm timber value chains in Mt Kenya area, Kenya

125. A Comparative financial analysis of current land use systems and implications for the adoption of improved agroforestry in the East Usambaras, Tanzania

126. Agricultural monitoring and evaluation systems

127. Challenges and opportunities for collaborative landscape governance in the East Usambara Mountains, Tanzania

128. Transforming Knowledge to Enhance Integrated Natural Resource Management Research, Development and Advocacy in the Highlands of Eastern Africa http://dx.doi.org/10.5716/WP11084.PDF

129. Carbon-forestry projects in the Philippines: potential and challenges The Mt Kitanglad Range forestcarbon development http://dx.doi.org10.5716/WP11054.PDF

130. Carbon forestry projects in the Philippines: potential and challenges. The Arakan Forest Corridor forest-carbon project. http://dx.doi.org10.5716/WP11055.PDF

131. Carbon-forestry projects in the Philippines: potential and challenges. The Laguna Lake Development Authority's forest-carbon development project. http://dx.doi.org/10.5716/WP11056.PDF

132. Carbon-forestry projects in the Philippines: potential and challenges. The Quirino forest-carbon development project in Sierra Madre Biodiversity Corridor http://dx.doi.org10.5716/WP11057.PDF

133. Carbon-forestry projects in the Philippines: potential and challenges. The Ikalahan Ancestral Domain forest-carbon development http://dx.doi.org10.5716/WP11058.PDF

134. The Importance of Local Traditional Institutions in the Management of Natural Resources in the Highlands of Eastern Africa. http://dx.doi.org/10.5716/WP11085.PDF 
135. Socio-economic assessment of irrigation pilot projects in Rwanda. http://dx.doi.org/10.5716/WP11086.PDF

136. Performance of three rambutan varieties (Nephelium lappaceum L.) on various nursery media. http://dx.doi.org/10.5716/WP11232.PDF

137. Climate change adaptation and social protection in agroforestry systems: enhancing adaptive capacity and minimizing risk of drought in Zambia and Honduras http://dx.doi.org/10.5716/WP11269.PDF

138. Does value chain development contribute to rural poverty reduction? Evidence of asset building by smallholder coffee producers in Nicaragua http://dx.doi.org/10.5716/WP11271.PDF

139. Potential for biofuel feedstock in Kenya. http://dx.doi.org/10.5716/WP11272.PDF

140. Impact of fertilizer trees on maize production and food security in six districts of Malawi. http://dx.doi.org/10.5716/WP11281.PDF

\section{2}

141. Fortalecimiento de capacidades para la gestión del Santuario Nacional Pampa Hermosa:

Construyendo las bases para un manejo adaptativo para el desarrollo local. Memorias del Proyecto. http://dx.doi.org/10.5716/WP12005.PDF

142. Understanding rural institutional strengthening: A cross-level policy and institutional framework for sustainable development in Kenya http://dx.doi.org/10.5716/WP12012.PDF

143. Climate change vulnerability of agroforestry http://dx.doi.org/10.5716/WP16722.PDF

144. Rapid assesment of the inner Niger delta of Mali http://dx.doi.org/10.5716/WP12021.PDF

145. Designing an incentive program to reduce on-farm deforestationin the East Usambara Mountains, Tanzania http://dx.doi.org/10.5716/WP12048.PDF

146. Extent of adoption of conservation agriculture and agroforestry in Africa: the case of Tanzania, Kenya, Ghana, and Zambia http://dx.doi.org/10.5716/WP12049.PDF

147. Policy incentives for scaling up conservation agriculture with trees in Africa: the case of Tanzania, Kenya, Ghana and Zambia http://dx.doi.org/10.5716/WP12050.PDF

148. Commoditized or co-invested environmental services? Rewards for environmental services scheme: River Care program Way Besai watershed, Lampung, Indonesia. http://dx.doi.org/10.5716/WP12051.PDF

149. Assessment of the headwaters of the Blue Nile in Ethiopia. http://dx.doi.org/10.5716/WP12160.PDF

150. Assessment of the uThukela Watershed, Kwazaulu. http://dx.doi.org/10.5716/WP12161.PDF

151. Assessment of the Oum Zessar Watershed of Tunisia. http://dx.doi.org/10.5716/WP12162.PDF

152. Assessment of the Ruwenzori Mountains in Uganda. http://dx.doi.org/10.5716/WP12163.PDF

153. History of agroforestry research and development in Viet Nam. Analysis of research opportunities and gaps. http://dx.doi.org/10.5716/WP12052.PDF

154. REDD+ in Indonesia: a Historical Perspective. http://dx.doi.org/10.5716/WP12053.PDF

155. Agroforestry and Forestry in Sulawesi series: Livelihood strategies and land use system dynamics in South Sulawesi http://dx.doi.org/10.5716/WP12054.PDF

156. Agroforestry and Forestry in Sulawesi series: Livelihood strategies and land use system dynamics in Southeast Sulawesi. http://dx.doi.org/10.5716/WP12055.PDF

157. Agroforestry and Forestry in Sulawesi series: Profitability and land-use systems in South and Southeast Sulawesi. http://dx.doi.org/10.5716/WP12056.PDF

158. Agroforestry and Forestry in Sulawesi series: Gender, livelihoods and land in South and Southeast Sulawesi http://dx.doi.org/10.5716/WP12057.PDF 
159. Agroforestry and Forestry in Sulawesi series: Agroforestry extension needs at the community level in AgFor project sites in South and Southeast Sulawesi, Indonesia. http://dx.doi.org/10.5716/WP12058.PDF

160. Agroforestry and Forestry in Sulawesi series: Rapid market appraisal of agricultural, plantation and forestry commodities in South and Southeast Sulawesi. http://dx.doi.org/10.5716/WP12059.PDF

\section{3}

161. Diagnosis of farming systems in the Agroforestry for Livelihoods of Smallholder farmers in Northwestern Viet Nam project http://dx.doi.org/10.5716/WP13033.PDF

162. Ecosystem vulnerability to climate change: a literature review. http://dx.doi.org/10.5716/WP13034.PDF

163. Local capacity for implementing payments for environmental services schemes: lessons from the RUPES project in northeastern Viet Nam http://dx.doi.org/10.5716/WP13046.PDF

164. Seri Agroforestri dan Kehutanan di Sulawesi: Agroforestry dan Kehutanan di Sulawesi: Strategi mata pencaharian dan dinamika sistem penggunaan lahan di Sulawesi Selatan http://dx.doi.org/10.5716/WP13040.PDF

165. Seri Agroforestri dan Kehutanan di Sulawesi: Mata pencaharian dan dinamika sistem penggunaan lahan di Sulawesi Tenggara http://dx.doi.org/10.5716/WP13041.PDF

166. Seri Agroforestri dan Kehutanan di Sulawesi: Profitabilitas sistem penggunaan lahan di Sulawesi Selatan dan Sulawesi Tenggara http://dx.doi.org/10.5716/WP13042.PDF

167. Seri Agroforestri dan Kehutanan di Sulawesi: Gender, mata pencarian dan lahan di Sulawesi Selatan dan Sulawesi Tenggara http://dx.doi.org/10.5716/WP13043.PDF

168. Seri Agroforestri dan Kehutanan di Sulawesi: Kebutuhan penyuluhan agroforestri pada tingkat masyarakat di lokasi proyek AgFor di Sulawesi Selatan dan Tenggara, Indonesia. http://dx.doi.org/10.5716/WP13044.PDF

169. Seri Agroforestri dan Kehutanan di Sulawesi: Laporan hasil penilaian cepat untuk komoditas pertanian, perkebunan dan kehutanan di Sulawesi Selatan dan Tenggara http://dx.doi.org/10.5716/WP13045.PDF

170. Agroforestry, food and nutritional security http://dx.doi.org/10.5716/WP13054.PDF

171. Stakeholder Preferences over Rewards for Ecosystem Services: Implications for a REDD+ Benefit Distribution System in Viet Nam http://dx.doi.org/10.5716/WP13057.PDF

172. Payments for ecosystem services schemes: project-level insights on benefits for ecosystems and the rural poor http://dx.doi.org/10.5716/WP13001.PDF

173. Good practices for smallholder teak plantations: keys to success http://dx.doi.org/10.5716/WP13246.PDF

174. Market analysis of selected agroforestry products in the Vision for Change Project intervention Zone, Côte d'Ivoire http://dx.doi.org/10.5716/WP13249.PDF

175. Rattan futures in Katingan: why do smallholders abandon or keep their gardens in Indonesia's 'rattan district'? http://dx.doi.org/10.5716/WP13251.PDF

176. Management along a gradient: the case of Southeast Sulawesi's cacao production landscapes http://dx.doi.org/10.5716/WP13265.PDF

177. Are trees buffering ecosystems and livelihoods in agricultural landscapes of the Lower Mekong Basin? Consequences for climate-change adaptation. http://dx.doi.org/10.5716/WP14047.PDF 
178. Agroforestry, livestock, fodder production and climate change adaptation and mitigation in East Africa: issues and options. http://dx.doi.org/10.5716/WP14050.PDF

179. Trees on farms: an update and reanalysis of agroforestry's global extent and socio-ecological characteristics. http://dx.doi.org/10.5716/WP14064.PDF

180. Beyond reforestation: an assessment of Vietnam's REDD+ readiness. http://dx.doi.org/10.5716/WP14097.PDF

181. Farmer-to-farmer extension in Kenya: the perspectives of organizations using the approach. http://dx.doi.org/10.5716/WP14380.PDF

182. Farmer-to-farmer extension in Cameroon: a survey of extension organizations. http://dx.doi.org/10.5716/WP14383.PDF

183. Farmer-to-farmer extension approach in Malawi: a survey of organizations: a survey of organizations http://dx.doi.org/10.5716/WP14391.PDF

184. Seri Agroforestri dan Kehutanan di Sulawesi: Kuantifikasi jasa lingkungan air dan karbon pola agroforestri pada hutan rakyat di wilayah sungai Jeneberang

185. Options for Climate-Smart Agriculture at Kaptumo Site in Kenyahttp://dx.doi.org/10.5716/WP14394.PDF

\section{5}

186. Agroforestry for Landscape Restoration and Livelihood Development in Central Asia http://dx.doi.org/10.5716/WP14143.PDF

187. "Projected Climate Change and Impact on Bioclimatic Conditions in the Central and South-Central Asia Region" http://dx.doi.org/10.5716/WP14144.PDF

188. Land Cover Changes, Forest Loss and Degradation in Kutai Barat, Indonesia. http://dx.doi.org/10.5716/WP14145.PDF

189. The Farmer-to-Farmer Extension Approach in Malawi: A Survey of Lead Farmers. http://dx.doi.org/10.5716/WP14152.PDF

190. Evaluating indicators of land degradation and targeting agroforestry interventions in smallholder farming systems in Ethiopia. http://dx.doi.org/10.5716/WP14252.PDF

191. Land health surveillance for identifying land constraints and targeting land management options in smallholder farming systems in Western Cameroon

192. Land health surveillance in four agroecologies in Malawi

193. Cocoa Land Health Surveillance: an evidence-based approach to sustainable management of cocoa landscapes in the Nawa region, South-West Côte d'Ivoire http://dx.doi.org/10.5716/WP14255.PDF

194. Situational analysis report: Xishuangbanna autonomous Dai Prefecture, Yunnan Province, China. http://dx.doi.org/10.5716/WP14255.PDF

195. Farmer-to-farmer extension: a survey of lead farmers in Cameroon. http://dx.doi.org/10.5716/WP15009.PDF

196. From transition fuel to viable energy source Improving sustainability in the sub-Saharan charcoal sector http://dx.doi.org/10.5716/WP15011.PDF

197. Mobilizing Hybrid Knowledge for More Effective Water Governance in the Asian Highlands http://dx.doi.org/10.5716/WP15012.PDF

198. Water Governance in the Asian Highlands http://dx.doi.org/10.5716/WP15013.PDF

199. Assessing the Effectiveness of the Volunteer Farmer Trainer Approach in Dissemination of Livestock Feed Technologies in Kenya vis-à-vis other Information Sources http://dx.doi.org/10.5716/WP15022.PDF

200. The rooted pedon in a dynamic multifunctional landscape: Soil science at the World Agroforestry Centre http://dx.doi.org/10.5716/WP15023.PDF 
201. Characterising agro-ecological zones with local knowledge. Case study: Huong Khe district, Ha Tinh, Viet Nam http://dx.doi.org/10.5716/WP15050.PDF

202. Looking back to look ahead: Insight into the effectiveness and efficiency of selected advisory approaches in the dissemination of agricultural technologies indicative of Conservation Agriculture with Trees in Machakos County, Kenya. http://dx.doi.org/10.5716/WP15065.PDF

203. Pro-poor Biocarbon Projects in Eastern Africa Economic and Institutional Lessons. http://dx.doi.org/10.5716/WP15022.PDF

204. Projected climate change impacts on climatic suitability and geographical distribution of banana and coffee plantations in Nepal. http://dx.doi.org/10.5716/WP15294.PDF

205. Agroforestry and Forestry in Sulawesi series: Smallholders' coffee production and marketing in Indonesia. A case study of two villages in South Sulawesi Province. http://dx.doi.org/10.5716/WP15690.PDF

206. Mobile phone ownership and use of short message service by farmer trainers: a case study of Olkalou and Kaptumo in Kenya http://dx.doi.org/10.5716/WP15691.PDF

207. Associating multivariate climatic descriptors with cereal yields: a case study of Southern Burkina Faso http://dx.doi.org/10.5716/WP15273.PDF

208. Preferences and adoption of livestock feed practices among farmers in dairy management groups in Kenya http://dx.doi.org/10.5716/WP15675.PDF

209. Scaling up climate-smart agriculture: lessons learned from South Asia and pathways for success http://dx.doi.org/10.5716/WP15720.PDF

210. Agroforestry and Forestry in Sulawesi series: Local perceptions of forest ecosystem services and collaborative formulation of reward mechanisms in South and Southeast Sulawesi http://dx.doi.org/10.5716/WP15721.PDF

211. Potential and challenges in implementing the co-investment of ecosystem services scheme in Buol District, Indonesia. http://dx.doi.org/10.5716/WP15722.PDF

212. Tree diversity and its utilization by the local community in Buol District, Indonesia http://dx.doi.org/10.5716/WP15723.PDF 


The World Agroforestry Centre is an autonomous, non-profit research organization whose vision is a rural transformation in the developing world as smallholder households increase their use of trees in agricultural landscapes to improve food security, nutrition, income, health, shelter, social cohesion, energy resources and environmental sustainability. The Centre generates science-based knowledge about the diverse roles that trees play in agricultural landscapes, and uses its research to advance policies and practices, and their implementation that benefit the poor and the environment. It aims to ensure that all this is achieved by enhancing the quality of its science work, increasing operational efficiency, building and maintaining strong partnerships, accelerating the use and impact of its research, and promoting greater cohesion, interdependence and alignment within the organization.

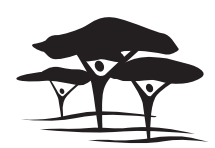

United Nations Avenue, Gigiri • PO Box 30677 • Nairobi, $00100 \cdot$ Kenya Telephone: +254 207224000 or via USA +1 6508336645 Fax: +254207224001 or via USA +16508336646 Email: worldagroforestry@cgiar.org•www.worldagroforestry.org

Southeast Asia Regional Program • Sindang Barang • Bogor 16680 PO Box $161 \cdot$ Bogor $16001 \cdot$ Indonesia

Telephone: +62 $2518625415 \cdot$ Fax: +62 2518625416 